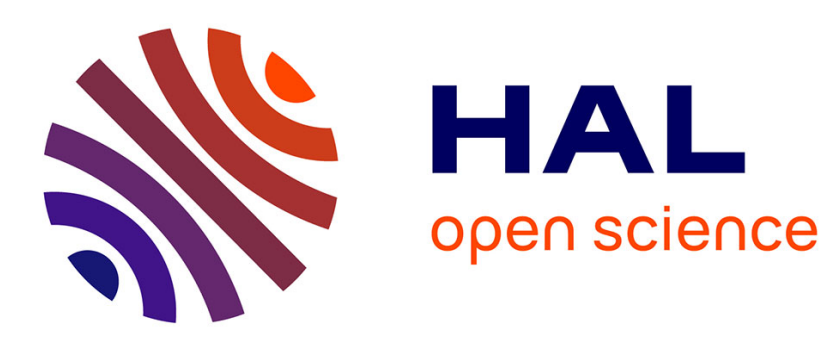

\title{
About the Lorentzian Yamabe problem
}

Nicolas Ginoux

\section{To cite this version:}

Nicolas Ginoux. About the Lorentzian Yamabe problem. Geometriae Dedicata, 2015, 174 (1), pp.287309. 10.1007/s10711-014-0018-8 . hal-01265984

\section{HAL Id: hal-01265984 https://hal.science/hal-01265984}

Submitted on 1 Feb 2016

HAL is a multi-disciplinary open access archive for the deposit and dissemination of scientific research documents, whether they are published or not. The documents may come from teaching and research institutions in France or abroad, or from public or private research centers.
L'archive ouverte pluridisciplinaire HAL, est destinée au dépôt et à la diffusion de documents scientifiques de niveau recherche, publiés ou non, émanant des établissements d'enseignement et de recherche français ou étrangers, des laboratoires publics ou privés. 


\title{
About the Lorentzian Yamabe problem
}

\author{
Nicolas Ginoux*
}

February 17, 2014

\begin{abstract}
We investigate the solutions to the Yamabe problem on globally hyperbolic spacetimes. On standard static spacetimes, we prove the existence of global solutions and show with the help of examples that uniqueness does not hold in general.
\end{abstract}

Mathematics Subject Classification (2010): 35L52, 35L70, 53B30, 53C50, 58J45

Keywords: Yamabe problem, Lorentzian geometry, globally hyperbolic spacetimes, nonlinear hyperbolic equations, Cauchy problem

\section{Introduction and summary of main results}

Let $\left(M^{n}, g\right)$ be an $n$-dimensional Lorentzian manifold with metric signature $(-+\ldots+)$. We want to solve the Yamabe problem on $\left(M^{n}, g\right)$, which is concerned with the existence of a metric with constant scalar curvature in the conformal class of $g$. We first recall its analytical formulation. Denote by $\square:=\delta_{g} \circ d=-\operatorname{tr}_{g}(\nabla \circ d)$ the scalar d'Alembert operator on $\left(M^{n}, g\right)$. If $S_{g}$ stands for the scalar curvature of $\left(M^{n}, g\right)$, then the transformation formulas for scalar curvature under conformal change of metric read respectively (see e.g. [8, Sec. 1.J])

$$
e^{2 u} S_{\bar{g}}=S_{g}+2 \square u
$$

for $n=2$ and $\bar{g}:=e^{2 u} g\left(\right.$ here $\left.u \in C^{\infty}(M, \mathbb{R})\right)$ and

$$
\frac{n-2}{4(n-1)} S_{\bar{g}} \varphi^{\frac{n+2}{n-2}}=\square \varphi+\frac{n-2}{4(n-1)} S_{g} \varphi
$$

for $n \geq 3$ and $\bar{g}:=\varphi^{\frac{4}{n-2}} g\left(\right.$ here $\left.\varphi \in C^{\infty}\left(M, \mathbb{R}_{+}^{\times}\right)\right)$.

From both identities above, the purely geometric question set by the Yamabe problem is equivalent to an analytical one, namely to solving (1) in dimension $n=2$ and $(2)$ in dimension $n \geq 3$ respectively: find a constant $S_{\bar{g}} \in \mathbb{R}$ such that a solution $u \in C^{\infty}(M, \mathbb{R})$ of 1 ( (resp. $\varphi \in C^{\infty}\left(M, \mathbb{R}_{+}^{\times}\right)$ of (2) ) exists.

Both (1) and (2) are semilinear (and nonlinear in case $S_{\bar{g}} \neq 0$ ) wave equations. Since such an equation can be locally put into the form of a symmetric hyperbolic system and such systems

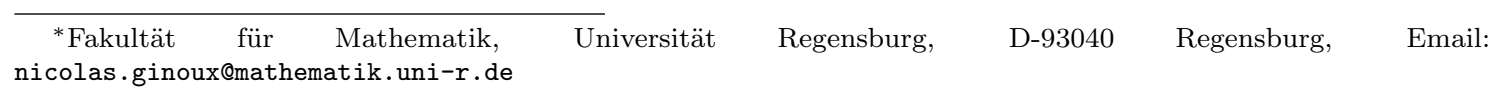


always have local smooth solutions (see e.g. [33, Ch. 16]), both (1) and (2) are locally solvable on any spacetime.

To investigate global existence (and possibly uniqueness) of solutions, it is reasonable to fix the geometric category of Lorentzian manifolds. We shall always assume $M$ to be endowed with a time-orientation (such Lorentzian manifolds are usually called spacetimes). Moreover, we shall mainly restrict ourselves to so-called globally hyperbolic spacetimes, which are diffeomorphic to the product of a spacelike hypersurface (called Cauchy hypersurface) with an interval, see Definition 2.1 below.

We first show (Theorem 2.4 that, for 2-dimensional globally hyperbolic spacetimes, the equation (1) always has a global solution for $S_{\bar{g}}=0$, whereas for $S_{\bar{g}} \neq 0$ only short-time-existence holds.

In higher dimensions, we focus on the case where a - hence any - Cauchy hypersurface of $M$ is closed (i.e., compact without boundary) and make a Cauchy-problem-ansatz, that is, we look for suitable values of the function and its first time derivative along a given Cauchy hypersurface such that the evolution equation (2) admits a global smooth positive solution on $M$. Due to local existence and uniqueness for solutions, given any initial data $\left(\varphi_{0}, \varphi_{1}\right)$ with $\varphi_{0}>0$ on some Cauchy hypersurface $\Sigma$, there exists in $M$ a maximal globally hyperbolic subdomain of existence for the solution $\varphi$ of the corresponding Cauchy problem with $\varphi_{\left.\right|_{\Sigma}}=\varphi_{0}$ and $\left.\frac{\partial \varphi}{\partial t}\right|_{\Sigma}=\varphi_{1}$ (Theorem 2.6. The proof of Theorem 2.6 requires a few geometric considerations about global hyperbolicity that we expose in detail to keep the article as self-contained as possible.

To see what kind of conditions can ensure the global existence of solutions, we first look for model examples and consider the simplest globally hyperbolic spacetimes at hand, namely the standard static ones. In that case, a separation of variables reduces (2) to a subcritical nonlinear eigenvalue equation on the Cauchy hypersurface (Proposition 3.3). In particular (2) always possesses a global solution on such spacetimes (Theorem 3.6) where, up to scale, the constant conformal scalar curvature coincides with the smallest eigenvalue of a Schrödinger operator on the Cauchy hypersurface. Next, we test the limits of this existence result by considering the question whether the constant conformal scalar curvature may be arbitrarily prescribed or not. Not surprisingly when compared to the Riemannian setting, the global existence of a solution to 2 with prescribed constant $S_{\bar{g}}$ depends on the sign of the above-mentioned smallest eigenvalue (Theorem 3.9p. However, as an unexpected consequence, the de Sitter spacetime is (globally) conformally scalar-flat only in dimensions up to 4 (Corollary 3.10).

Still in the framework of standard static spacetimes, we address the question of uniqueness of solutions. Although we cannot presently make any general statement, for numerous situations we consider in Section 3.2 - in particular the case where the scalar curvature of the spacetime is constant negative - uniqueness never holds, see Corollary 3.13. Theorem 3.14 and Example 3.15. We conjecture that the solutions of the Yamabe equation are never unique.

We conclude by discussing briefly the difficulties we face when coming back to arbitrary globally hyperbolic spacetimes. We insist on the fact that the present paper should be seen as a first step in solving the Yamabe problem and that there is still a lot to be done to fully understand the globally hyperbolic setting.

This article is based on [14, Ch. 5]. 


\section{Preliminaries and the 2-dimensional case}

In this section, we recall the main concepts we need throughout the paper and treat the 2dimensional case. The first notion to be introduced is that of global hyperbolicity.

Definition 2.1 A spacetime $\left(M^{n}, g\right)$ is called globally hyperbolic if and only if there exists a Cauchy hypersurface in $M$, that is, a subset $\Sigma$ of $M$ which is met exactly once by every inextendible timelike curve in $M$.

By [7, Thm. 3.2], a spacetime is globally hyperbolic if and only if it has no closed (future- or past-directed) causal curve and all subsets of the form $J_{+}^{M}(p) \cap J_{-}^{M}(q), p, q \in M$, are compact. If $\Sigma$ is a smooth spacelike Cauchy hypersurface of $M$, then actually it is met exactly once by any inextendible causal curve in $M$. We also recall the following smooth splitting theorem for globally hyperbolic spacetimes:

Theorem 2.2 (A. Bernal \& M. Sánchez [5, 6]) Let $\left(M^{n}, g\right)$ be a spacetime.

i) If $\left(M^{n}, g\right)$ is globally hyperbolic, then it is isometric to $\left(\mathbb{R} \times \Sigma,-\beta d t^{2} \oplus g_{t}\right)$, where each $\{t\} \times \Sigma$ corresponds to a smooth spacelike Cauchy hypersurface of $M, \beta \in C^{\infty}\left(\mathbb{R} \times \Sigma, \mathbb{R}_{+}^{\times}\right)$and $\left(g_{t}\right)_{t}$ is a smooth 1-parameter family of Riemannian metrics on $\Sigma$.

ii) If $\Sigma \subset M$ is any given smooth spacelike Cauchy hypersurface in the (globally hyperbolic) spacetime $\left(M^{n}, g\right)$, then for any $t_{0} \in \mathbb{R}$ there is an isometry $\left(M^{n}, g\right) \cong\left(\mathbb{R} \times \Sigma,-\beta d t^{2} \oplus g_{t}\right)$ as above and where $\Sigma$ identifies with $\left\{t_{0}\right\} \times \Sigma$.

For instance, the warped product $(M, g)=\left(I \times \Sigma,-d t^{2} \oplus b(t)^{2} g_{\Sigma}\right)$ of an open interval $I \subset \mathbb{R}$ with a Riemannian manifold $\left(\Sigma, g_{\Sigma}\right)$ (where $b \in C^{\infty}\left(I, \mathbb{R}_{+}^{\times}\right)$is arbitrary) is globally hyperbolic if and only if $\left(\Sigma, g_{\Sigma}\right)$ is complete, see e.g. [4, Thm. 3.66] or [3, Lemma A.5.14]. This class contains for instance all Robertson-Walker spacetimes, in particular the Minkowski and the de Sitter spacetimes. It is however important to note that, in general, Theorem 2.2 only implies the existence of a smooth splitting in the form $\left(\mathbb{R} \times \Sigma,-\beta d t^{2} \oplus g_{t}\right)$, and that the induced Riemannian metric $g_{t}$ on $\Sigma$ need not be complete, see e.g. [1, Sec. 2.5] for concrete examples. Let us also mention that any product of the form $\left(I \times \Sigma,-\beta d t^{2} \oplus g_{t}\right)$ with closed $\Sigma$ is globally hyperbolic and that every $\{t\} \times \Sigma$ is a Cauchy hypersurface in $M$ [29, Cor. 3.3].

Since the causal type for vectors does not change when rescaling pointwise the metric, it is easy to see that $\left(M^{n}, g\right)$ is globally hyperbolic if and only if $\left(M^{n}, \bar{g}\right)$ is globally hyperbolic, for any metric $\bar{g}$ conformal to $g$. By conformal invariance of the Yamabe problem, we can therefore - and will in most cases - assume that $\beta=1$, that is, that $g=-d t^{2} \oplus g_{t}$ on $I \times \Sigma$. Before studying the above equations in particular cases, we give the following useful formulas, which can be proved in an elementary way:

Lemma 2.3 Let a spacetime $\left(M^{n}, g\right)$ be of the form $\left(I \times \Sigma,-\beta d t^{2} \oplus g_{t}\right)$ where $\beta \in C^{\infty}\left(I \times \Sigma, \mathbb{R}_{+}^{\times}\right)$ and $\left(g_{t}\right)_{t}$ is a smooth 1-parameter family of Riemannian metrics on $\Sigma$. Then the following identities hold.

1. For every $f \in C^{\infty}(M, \mathbb{R})$,

$$
\begin{aligned}
\square f= & \frac{1}{\beta} \frac{\partial^{2} f}{\partial t^{2}}+\frac{1}{2 \beta}\left(\operatorname{tr}_{g_{t}}\left(\frac{\partial g_{t}}{\partial t}\right)-\frac{1}{\beta} \frac{\partial \beta}{\partial t}\right) \frac{\partial f}{\partial t} \\
& -\frac{1}{2 \beta} g_{t}\left(\operatorname{grad}_{g_{t}}(\beta(t, \cdot)), \operatorname{grad}_{g_{t}}(f(t, \cdot))\right)+\Delta_{g_{t}} f(t, \cdot),
\end{aligned}
$$

where $\Delta_{g_{t}}:=\delta_{g_{t}}^{\Sigma} \circ d=-\operatorname{tr}_{g_{t}}\left(\operatorname{Hess}_{g_{t}}^{\Sigma}(\cdot)\right): C^{\infty}(\Sigma, \mathbb{R}) \rightarrow C^{\infty}(\Sigma, \mathbb{R})$. 
2. In case $\beta=1$, we have

$$
\square=\frac{\partial^{2}}{\partial t^{2}}+\frac{1}{2} \operatorname{tr}_{g_{t}}\left(\frac{\partial g_{t}}{\partial t}\right) \frac{\partial}{\partial t}+\Delta_{g_{t}} .
$$

3. In case $\beta=1$ and $g_{t}=b(t)^{2} g_{\Sigma}$ for some $b \in C^{\infty}\left(I, \mathbb{R}_{+}^{\times}\right)$and some Riemannian metric $g_{\Sigma}$ on $\Sigma$, one has

$$
\begin{aligned}
\square+a_{n} S_{g}= & \frac{\partial^{2}}{\partial t^{2}}+(n-1) \frac{b^{\prime}}{b} \frac{\partial}{\partial t}+\frac{1}{b^{2}} \Delta_{g_{\Sigma}} \\
& +\frac{a_{n}}{b^{2}}\left(S_{g_{\Sigma}}+2(n-1) b b^{\prime \prime}+(n-1)(n-2)\left(b^{\prime}\right)^{2}\right),
\end{aligned}
$$

where $a_{n}:=\frac{n-2}{4(n-1)}$ and where $S_{g}$ and $S_{g_{\Sigma}}$ are the scalar curvatures of $(M, g)$ and $\left(\Sigma, g_{\Sigma}\right)$ respectively.

4. In case $\beta=1$ and $g_{t}=g_{\Sigma}$ for some Riemannian metric $g_{\Sigma}$ on $\Sigma$, one has

$$
\square+a_{n} S_{g}=\frac{\partial^{2}}{\partial t^{2}}+L_{g_{\Sigma}}
$$

where $L_{g_{\Sigma}}:=\Delta_{g_{\Sigma}}+a_{n} S_{g_{\Sigma}}$.

We first deal with the case $n=2$. The following theorem can be seen as the 2-dimensional analogue of Theorem 3.9 below.

Theorem 2.4 Let $\left(M^{2}, g\right)$ be a connected 2-dimensional globally hyperbolic spacetime.

1) Then $\left(M^{2}, g\right)$ is globally conformally flat, i.e., (1) with $S_{\bar{g}}=0$ always has a global smooth solution on $M$.

2) If $S_{\bar{g}} \in \mathbb{R}^{\times}$, then there is no solution to 1 on $\left(\mathbb{R} \times \mathbb{S}^{1},-d t^{2} \oplus d s^{2}\right)$.

Proof: Theorem 2.2 yields a smooth splitting $\left(M^{2}, g\right)=\left(I \times \Sigma,-\beta d t^{2} \oplus g_{t}\right)$, where $I \subset \mathbb{R}$ is an open interval, $\beta \in C^{\infty}\left(M, \mathbb{R}_{+}^{\times}\right)$, each $\{t\} \times \Sigma$ is a smooth spacelike Cauchy hypersurface in $M$ and $\left(g_{t}\right)_{t \in I}$ is a smooth one-parameter family of Riemannian metrics on $\Sigma$. Since (1) with $S_{\bar{g}}=0$ is a linear wave equation, we may solve directly the Cauchy problem associated to it: fixing $t_{0} \in I$, a future unit normal $\nu$ along $\left\{t_{0}\right\} \times \Sigma$ and $u_{0}, u_{1} \in C^{\infty}(\Sigma, \mathbb{R})$, the Cauchy problem with smooth (but non-necessarily compactly-supported) data $\square u=-\frac{S_{g}}{2}, u_{\left.\right|_{\left\{t_{0}\right\} \times \Sigma}}=u_{0}, \partial_{\nu} u_{\left.\right|_{\left\{t_{0}\right\} \times \Sigma}}=u_{1}$ is linear (inhomogeneous), hence always solvable on globally hyperbolic spacetimes, see e.g. [13, Cor. 5]. This shows 1 ).

Let $S_{\bar{g}} \in \mathbb{R}^{\times}$be arbitrary. Assume the existence of $u \in C^{\infty}\left(\mathbb{R} \times \mathbb{S}^{1}, \mathbb{R}\right)$ solving $(1)$, i.e., $\square u=\frac{S_{\bar{g}}}{2} e^{2 u}$ on $\mathbb{R} \times \mathbb{S}^{1}$. Setting $y: \mathbb{R} \rightarrow \mathbb{R}, t \mapsto \int_{\mathbb{S}^{1}} u(t, x) d x$, the function $y$ is smooth with

$$
\begin{aligned}
y^{\prime \prime}(t) & =\int_{\mathbb{S}^{1}} \frac{\partial^{2} u}{\partial t^{2}}(t, x) d x \\
& =\int_{\mathbb{S}^{1}}(\square u)(t, x) d x \quad \text { since } \int_{\mathbb{S}^{1}} \frac{\partial^{2} u}{\partial x^{2}}(t, x) d x=0 \\
& =\frac{S_{\bar{g}}}{2} \int_{\mathbb{S}^{1}} e^{2 u(t, x)} d x,
\end{aligned}
$$

compare with the proof of Theorem 3.9 below. Assume $S_{\bar{g}}>0$. Denoting by $L>0$ the length of $\mathbb{S}^{1}$, Jensen's inequality yields

$$
y^{\prime \prime} \geq \frac{S_{\bar{g}} L}{2} \exp \left(\frac{1}{L} \int_{\mathbb{S}^{1}} 2 u(t, x) d x\right)=\frac{S_{\bar{g}} L}{2} e^{\frac{2 y}{L}}
$$


on $\mathbb{R}$. But no function satisfying that differential inequality can exist on $\mathbb{R}$, see also the proof of Theorem 3.9 below. Namely, up to replacing $y$ by $t \mapsto y(\alpha t)$ for a suitable $\alpha \in \mathbb{R}_{+}^{\times}$, we assume that $y$ satisfies $y^{\prime \prime} \geq \frac{1}{2} e^{\frac{2 y}{L}}$. Since in particular $y$ is strictly convex, we may assume up to changing $t$ into $\pm t+t_{0}$ for a constant $t_{0} \in \mathbb{R}$ that $y^{\prime} \geq 0$ on $\left[0, \infty\left[\right.\right.$. Multiplying with $y^{\prime}$ yields $y^{\prime \prime} y^{\prime} \geq \frac{y^{\prime}}{2} e^{\frac{2 y}{L}}$ so that $\left(y^{\prime}\right)^{2}(t)-\left(y^{\prime}\right)^{2}(0) \geq \frac{L}{2}\left(e^{\frac{2 y(t)}{L}}-e^{\frac{2 y(0)}{L}}\right)$ for every $t \geq 0$, which in turn gives

$$
\int_{y(0)}^{y(t)} \frac{d z}{\sqrt{e^{\frac{2 z}{L}}-e^{\frac{2 y(0)}{L}}}} \geq \frac{L}{2} t
$$

for every $t \geq 0$. Because of $\int_{y(0)}^{\infty} \frac{d z}{\sqrt{e^{\frac{2 z}{L}}-e^{\frac{2 y(0)}{L}}}}<\infty$, the existence interval of $y$ is bounded above, or in other words $y(t) \rightarrow \infty$ in finite time. In particular, $y$ is not defined on $\mathbb{R}$. The case where $S_{\bar{g}}<0$ is analogous (this time $y$ is concave and goes to $-\infty$ in finite time). This shows 2) and concludes the proof.

\section{Notes 2.5}

1. Since the Cauchy data for (1) along a given Cauchy hypersurface may be prescribed arbitrarily, there are actually infinitely many conformal flat metrics which are non homothetic to each other on a given globally hyperbolic 2-dimensional spacetime. Alternatively - and as is well-known - all solutions to $\square u=0$ on $\left(M^{2}, g\right)=\left(I \times \Sigma,-d t^{2} \oplus d s^{2}\right)$ are of the form $u(t, s)=v(t+s)+w(t-s)$, with arbitrary (and periodic if $\Sigma=\mathbb{S}^{1}$ ) smooth functions $v, w$ on $\mathbb{R}$, see also Note $3.7,2$ below.

2. For $S_{\bar{g}} \in \mathbb{R}^{\times}$, Theorem 2.4 states that there is no solution to 1 on $M^{2}=I \times \mathbb{S}^{1}$ when the time interval $I$ is long enough. But solutions exist for short $I$, as we know anyway from the local theory mentioned above. For example, the 2-dimensional de Sitter spacetime, which can be described as the warped product $\left(\mathbb{R} \times \mathbb{S}^{1},-d t^{2} \oplus \cosh (t)^{2} d s^{2}\right)$, is conformally equivalent to the flat cylinder (]$-\frac{\pi}{2}, \frac{\pi}{2}\left[\times \mathbb{S}^{1},-d t^{2} \oplus d s^{2}\right)$, see Corollary 3.10 below. In particular, there exists a conformal metric with scalar curvature 2 on (]$-\frac{\pi}{2}, \frac{\pi}{2}\left[\times \mathbb{S}^{1},-d t^{2} \oplus d s^{2}\right)$.

In the non globally hyperbolic setting, conformal flatness may or may not hold. For instance, the 2-dimensional anti de Sitter spacetime $\left(\mathbb{S}^{1} \times \mathbb{S}_{+}^{1}, \frac{1}{x_{2}^{2}}\left(-d t^{2} \oplus d s^{2}\right)\right)$ (where $\left(x_{1}, x_{2}\right)$ are the cartesian coordinates for the second factor $\left.\mathbb{S}_{+}^{1}:=\left\{\left(x_{1}, x_{2}\right) \in \mathbb{S}^{1} \mid x_{2}>0\right\}\right)$ is obviously conformally flat. On $M=\mathbb{R}^{2}$ or the 2-torus $\mathbb{T}^{2}$, Miguel Sánchez has shown that an arbitrary metric $g$ is conformally flat if and only if it admits a non-zero conformal Killing vector field which is everywhere timelike or everywhere spacelike [28, Thm. 2.3]. Moreover, he constructed whole families of metrics on $\mathbb{T}^{2}$ (and $\mathbb{R}^{2}$ ) without any such conformal Killing vector field and which hence are not conformally flat [28, Sec. 3]. Note that none of those metrics on $\mathbb{R}^{2}$ can be globally hyperbolic by Theorem 2.4 .

Let us mention that there is still a lot of freedom left when prescribing scalar curvature functions in 2 dimensions: generalizing previous work by John Burns [10, Thm. 2.2], Marc Nardmann proved that any function which is either identically vanishing or sign-changing on a closed Lorentzian surface $M$ is the scalar curvature of some Lorentzian metric on $M$ [25, Thm. 1.3.13].

From now on, we assume $n \geq 3$. In that case we know local solutions exist by the remarks above. One can do a bit better: as for the existence problem for solutions to the Einstein equations [11, Thm. 3], there is a maximal domain of existence for solutions to the Yamabe problem: 
Theorem 2.6 Let $\left(M^{n}, g\right)$ be an $n(\geq 3)$-dimensional globally hyperbolic spacetime with smooth spacelike closed Cauchy hypersurface $\Sigma \subset M$ and $S_{\bar{g}} \in \mathbb{R}$ be an arbitrary constant. Denote by $\nu \in \Gamma\left(T^{\perp} \Sigma\right)$ the future-directed (timelike) unit normal along $\Sigma$. Then for any $\varphi_{0}, \varphi_{1} \in C^{\infty}(\Sigma, \mathbb{R})$ with $\varphi_{0}>0$, there exists a unique maximal globally hyperbolic open subset $\widehat{D}_{\Sigma}$ of $M$ in which $\Sigma$ is a Cauchy hypersurface and on which the Cauchy problem (2) with $\varphi_{\left.\right|_{\Sigma}}=\varphi_{0}$ and $\partial_{\nu} \varphi=\varphi_{1}$ has a unique smooth positive solution.

Proof: The proof mainly relies on local existence and (global) uniqueness for solutions to the Cauchy problem

$$
\begin{cases}\square \varphi+a_{n} S_{g} \varphi & =a_{n} S_{\bar{g}} \varphi^{\frac{n+2}{n-2}} \\ \varphi_{\mid \Sigma} & =\varphi_{0} \\ \partial_{\nu} \varphi & =\varphi_{1},\end{cases}
$$

which both follow from the theory of symmetric hyperbolic systems. Namely for any $\varphi_{0}, \varphi_{1} \in$ $C^{\infty}(\Sigma, \mathbb{R})$ with $\varphi_{0}>0$ consider the set

$$
\begin{aligned}
\mathcal{M}_{\Sigma, \varphi_{0}, \varphi_{1}}:= & \left\{D_{\Sigma} \subset M, D_{\Sigma} \text { open, } \Sigma \text { Cauchy hypersurface of } D_{\Sigma},\right. \\
& \left.\exists \varphi \in C^{\infty}\left(D_{\Sigma}, \mathbb{R}_{+}^{\times}\right) \text {solving }[7] \text { on } D_{\Sigma}\right\} .
\end{aligned}
$$

Note that, by uniqueness of solutions to symmetric hyperbolic systems, for each $D_{\Sigma} \in \mathcal{M}_{\Sigma, \varphi_{0}, \varphi_{1}}$, there is a unique positive smooth solution $\varphi$ to (2) on $D_{\Sigma}$ with Cauchy data $\varphi_{0}, \varphi_{1}$. Local existence for the Cauchy problem along the compact Cauchy hypersurface $\Sigma$ already ensures $\mathcal{M}_{\Sigma, \varphi_{0}, \varphi_{1}} \neq \varnothing$ : if $\left(M^{n}, g\right)=\left(\mathbb{R} \times \Sigma,-\beta d t^{2} \oplus g_{t}\right)$ is split as in Theorem 2.2, where say $\Sigma \simeq\{0\} \times \Sigma$, then there is a nonempty open interval $J \subset \mathbb{R}$ about 0 for which a smooth positive solution to the Cauchy problem (7) exists on the open subset $J \times \Sigma$ of $M$; but with the induced metric and time orientation, $J \times \Sigma$ is clearly globally hyperbolic with $\Sigma$ as a Cauchy hypersurface, therefore $J \times \Sigma \in \mathcal{M}_{\Sigma, \varphi_{0}, \varphi_{1}}$.

Next define $\widehat{D}_{\Sigma}:=\bigcup_{D_{\Sigma} \in \mathcal{M}_{\Sigma, \varphi_{0}, \varphi_{1}}} D_{\Sigma} \subset M$, which is open in $M$ and contains $\Sigma$. We claim that $\widehat{D}_{\Sigma} \in \mathcal{M}_{\Sigma, \varphi_{0}, \varphi_{1}}$. First, we show that $\Sigma$ is a Cauchy hypersurface of $\widehat{D}_{\Sigma}$ (hence $\widehat{D}_{\Sigma}$ is globally hyperbolic). The proof of this is based on the following two claims.

Claim 1: Let $\Omega \subset M$ be any nonempty open subset which is causally compatible in $M$ (for any $\left.p \in \Omega, J_{ \pm}^{M}(p) \cap \Omega=J_{ \pm}^{\Omega}(p)\right)$. Then $\Omega$ itself - with the induced metric and time orientation - is globally hyperbolic if and only if $J_{+}^{M}(p) \cap J_{-}^{M}(q) \subset \Omega$ for all $p, q \in \Omega$.

Proof of Claim 1: There exists no closed causal curve in $\Omega$ since there is already none in $M$. If $\Omega$ is globally hyperbolic, then for all $p, q \in \Omega$ the subset $J_{+}^{\Omega}(p) \cap J_{-}^{\Omega}(q)$ is compact; but by causal compatibility of $\Omega, J_{+}^{\Omega}(p) \cap J_{-}^{\Omega}(q)=J_{+}^{M}(p) \cap J_{-}^{M}(q) \cap \Omega$; now $J_{+}^{M}(p) \cap J_{-}^{M}(q)$ is by construction (path-)connected, so that the intersection $J_{+}^{M}(p) \cap J_{-}^{M}(q) \cap \Omega$, being open and closed in $J_{+}^{M}(p) \cap J_{-}^{M}(q)$, is either empty or the whole subset $J_{+}^{M}(p) \cap J_{-}^{M}(q)$; in the first case, necessarily $J_{+}^{M}(p) \cap J_{-}^{M}(q)=\varnothing$ holds (otherwise $q \in J_{+}^{M}(p) \cap J_{-}^{M}(q) \cap \Omega$ ) and hence $J_{+}^{\Omega}(p) \cap J_{-}^{\Omega}(q)=$ $J_{+}^{M}(p) \cap J_{-}^{M}(q)$; in the second case, we also obtain $J_{+}^{\Omega}(p) \cap J_{-}^{\Omega}(q)=J_{+}^{M}(p) \cap J_{-}^{M}(q)$. In both cases, we have $J_{+}^{M}(p) \cap J_{-}^{M}(q) \subset \Omega$. Conversely, if $J_{+}^{M}(p) \cap J_{-}^{M}(q) \subset \Omega$ for all $p, q \in \Omega$, then $J_{+}^{\Omega}(p) \cap J_{-}^{\Omega}(q)=J_{+}^{M}(p) \cap J_{-}^{M}(q) \cap \Omega=J_{+}^{M}(p) \cap J_{-}^{M}(q)$ is compact for all $p, q \in \Omega$ and thus $\Omega$ is globally hyperbolic.

Claim 2: If $\Sigma$ is a Cauchy hypersurface of an open subset $\Omega \subset M$, then $\Omega$ is automatically causally compatible in $M$.

Proof of Claim 2: Let $p \in \Omega$ and $q \in J_{+}^{M}(p) \cap \Omega$ be arbitrary. Pick a future-directed causal curve $c:[0,1] \rightarrow M$ with $c(0)=p$ and $c(1)=q$ in $M$ and extend it to an inextendible futuredirected causal curve $\widetilde{c}: \mathbb{R} \rightarrow M$. We consider the following cases. First, let $p \in J_{+}^{\Omega}(\Sigma)$. Since $\Sigma$ is a spacelike Cauchy hypersurface of $M$, there exists a unique $t_{0} \in \mathbb{R}$ with $\widetilde{c}\left(t_{0}\right) \in \Sigma$; note that $t_{0} \leq 0$ because of $p \in J_{+}^{\Omega}(\Sigma) \subset J_{+}^{M}(\Sigma) \cap \Omega$. Define $t_{\min }:=\inf \{t<1 \mid \widetilde{c}(s) \in \Omega \forall s \in[t, 1]\}$ 
and $t_{\max }:=\sup \{t>1 \mid \widetilde{c}(s) \in \Omega \forall s \in[1, t]\}$. Note that $t_{\min } \in\left[-\infty, 1\left[\right.\right.$ and $\left.\left.t_{\max } \in\right] 1, \infty\right]$ are well-

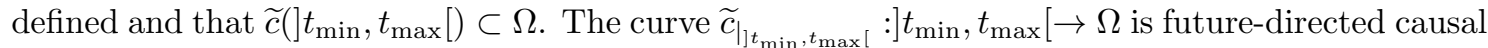
and inextendible as a curve in $\Omega$ by construction of $t_{\min }$ and $t_{\max }$, therefore it meets the Cauchy hypersurface $\Sigma$ of $\Omega$ in exactly one point. But since $t_{0}$ is the unique $t \in \mathbb{R}$ with $\widetilde{c}(t) \in \Sigma$, one necessarily has $t_{\min }<t_{0}$, in particular $t_{\min }<0$, from which $\widetilde{c}(s)=c(s) \in \Omega$ for all $\left.s \in[0,1] \subset\right] t_{\min }, t_{\max }[$ follows. This implies $q \in J_{+}^{\Omega}(p)$. The case where $q \in J_{-}^{\Omega}(\Sigma)$ is analogous (just "reverse" time). The last case where $p$ and $q$ are on two different sides of $\Sigma$ (i.e., $p \in I_{-}^{\Omega}(\Sigma)$ and $q \in I_{+}^{\Omega}(\Sigma)$ ) is also similar: one may assume $c\left(\frac{1}{2}\right) \in \Sigma$ and then one shows as above that both restrictions $c_{\left[0, \frac{1}{2}\right]}$ and $c_{\left[\frac{1}{2}, 1\right]}$ run entirely in $\Omega$. Therefore $q \in J_{+}^{\Omega}(p)$ in all three cases. Obviously $J_{+}^{\Omega}(p) \subset J_{+}^{M}(p) \cap \Omega$ always holds true, thus we have shown $J_{+}^{\Omega}(p)=J_{+}^{M}(p) \cap \Omega$ for all $p \in \Omega$. Reversing time we also show $J_{-}^{\Omega}(p)=J_{-}^{M}(p) \cap \Omega$ for all $p \in \Omega$ and hence $\Omega$ is causally compatible.

To show that $\Sigma$ is a Cauchy hypersurface of $\widehat{D}_{\Sigma}$, let $c: \mathbb{R} \rightarrow \widehat{D}_{\Sigma}$ be any inextendible future-directed timelike curve. Then its intersection with each $D_{\Sigma}$ - that we denote by $c \cap D_{\Sigma}$ - is again a curve (and remains inextendible, timelike and future-directed): for any $s \leq t \in \mathbb{R}$ with $c(s), c(t) \in D_{\Sigma}$, one has $c(u) \in J_{+}^{M}(c(s)) \cap J_{-}^{M}(c(t))$ for all $u \in[s, t]$ and, because $\bar{D}_{\Sigma}$ is causally compatible by Claim 2, we have $J_{+}^{M}(c(s)) \cap J_{-}^{M}(c(t)) \subset D_{\Sigma}$ by Claim 1 and hence $c(u) \in D_{\Sigma}$. Therefore $c \cap D_{\Sigma}$ meets $\Sigma$ in (exactly) one point, from which follows that $c$ meets $\Sigma$ in one point, which must be unique since $\Sigma$ can anyway be met only once by causal curves. Therefore $\Sigma$ is a Cauchy hypersurface of $\widehat{D}_{\Sigma}$.

It remains to show the existence of a $\varphi \in C^{\infty}\left(\widehat{D}_{\Sigma}, \mathbb{R}_{+}^{\times}\right)$solving $\left.\sqrt{7}\right)$ on $\widehat{D}_{\Sigma}$. For this, we first show that $\mathcal{M}_{\Sigma, \varphi_{0}, \varphi_{1}}$ is stable under finite intersection. For any $D_{\Sigma}^{1}, D_{\Sigma}^{2} \in \mathcal{M}_{\Sigma, \varphi_{0}, \varphi_{1}}$, consider any inextendible timelike curve $c$ in $D_{\Sigma}^{1} \cap D_{\Sigma}^{2}$. Then one can extend $c$ to inextendible causal curves $\widetilde{c}^{i}$ in $D_{\Sigma}^{i}, i=1,2$ (of course it may happen that one - or both - extension already coincides with $c$ itself), each of which meets $\Sigma$ in exactly one point. Gluing $\widetilde{c}^{1}$ with $\widetilde{c}^{2}$ along $c$ one obtains a future-directed causal curve $\widetilde{c}$ in $D_{\Sigma}^{1} \cup D_{\Sigma}^{2}$ - this is a (piecewise smooth) curve since no two extensions can come out of the same end of $c$ unless $c$ is already extendible - which is also inextendible in $D_{\Sigma}^{1} \cup D_{\Sigma}^{2}$. By the above argument (applicable to any union of elements of $\mathcal{M}_{\Sigma, \varphi_{0}, \varphi_{1}}$ ), $\Sigma$ is a Cauchy hypersurface of $D_{\Sigma}^{1} \cup D_{\Sigma}^{2}$, therefore $\widetilde{c}$ meets $\Sigma$ in exactly one point, which by uniqueness must lie in both $D_{\Sigma}^{1}$ and $D_{\Sigma}^{2}$; in turn this implies that $c$ meets $\Sigma$ in exactly one point. Therefore $\Sigma$ is a Cauchy hypersurface in $D_{\Sigma}^{1} \cap D_{\Sigma}^{2}$. It remains to notice that the solutions $\varphi^{1}$ and $\varphi^{2}$ to (7) on $D_{\Sigma}^{1}$ and $D_{\Sigma}^{2}$ respectively have to coincide on $D_{\Sigma}^{1} \cap D_{\Sigma}^{2}$ by uniqueness of solutions to (7) on the globally hyperbolic spacetime $D_{\Sigma}^{1} \cap D_{\Sigma}^{2}$. Therefore $D_{\Sigma}^{1} \cap D_{\Sigma}^{2} \in \mathcal{M}_{\Sigma, \varphi_{0}, \varphi_{1}}$.

Coming back to the Cauchy problem on $\widehat{D}_{\Sigma}$, define $\varphi$ on $\widehat{D}_{\Sigma}$ via $\varphi(p):=\varphi^{i}(p)$ for $p \in D_{\Sigma}^{i}$, where $\varphi^{i} \in C^{\infty}\left(D_{\Sigma}^{i}, \mathbb{R}_{+}^{\times}\right)$solves [7] on $D_{\Sigma}^{i}$; since $D_{\Sigma}^{i} \cap D_{\Sigma}^{j} \in \mathcal{M}_{\Sigma, \varphi_{0}, \varphi_{1}}$ for any $D_{\Sigma}^{i}, D_{\Sigma}^{j} \in \mathcal{M}_{\Sigma, \varphi_{0}, \varphi_{1}}$, we have $\left.\varphi^{i}\right|_{D_{\Sigma}^{i} \cap D_{\Sigma}^{j}}=\left.\varphi^{j}\right|_{D_{\Sigma}^{i} \cap D_{\Sigma}^{j}}$, so that the function $\varphi$ is well-defined, positive, smooth and solves 17 on $\widehat{D}_{\Sigma}$. This shows $\widehat{D}_{\Sigma} \in \mathcal{M}_{\Sigma, \varphi_{0}, \varphi_{1}}$. By construction, $\widehat{D}_{\Sigma}$ is maximal and is unique since it contains every element of $\mathcal{M}_{\Sigma, \varphi_{0}, \varphi_{1}}$. This concludes the proof of Theorem 2.6.

Of course, the maximal domain $\widehat{D}_{\Sigma}$ of Theorem 2.6 depends on $\Sigma$, on the metric $g$, on $S_{\bar{g}}$ and on the Cauchy data $\varphi_{0}, \varphi_{1}$. Note also that the analogous statement from Theorem 2.6 also holds true in dimension 2 for the Cauchy problem corresponding to (1). In the next sections, we discuss when $\widehat{D}_{\Sigma}=M$ for $M$ in a particular subcategory of spacetimes.

\section{Conformally standard static spacetimes}

In this section, we start with the particular case where $\left(M^{n}, g\right)$ is conformally equivalent to the product $\left(I \times \Sigma,-d t^{2} \oplus g_{\Sigma}\right)$ of an open interval $I \subset \mathbb{R}$ with a closed Riemannian manifold $\left(\Sigma^{n-1}, g_{\Sigma}\right)$. 
Following the literature, products are a particular case of so-called standard static spacetimes:

Definition 3.1 A spacetime $\left(M^{n}, g\right)$ is called

i) static if and only if it admits a timelike Killing vector field whose orthogonal distribution is integrable.

ii) standard static if and only if it is isometric to a product $\left(I \times \Sigma,-\beta d t^{2} \oplus g_{\Sigma}\right)$ for some open interval $I \subset \mathbb{R}$, some Riemannian manifold $\left(\Sigma^{n-1}, g_{\Sigma}\right)$ and some $\beta \in C^{\infty}\left(\Sigma, \mathbb{R}_{+}^{\times}\right)$.

Any standard static spacetime is static (take e.g. $\frac{\partial}{\partial t}$ as timelike Killing vector field with integrable orthogonal distribution) and any static spacetime is locally standard static. A simply connected static spacetime $\left(M^{n}, g\right)$ is standard static if and only if at least one of its static vector fields (Killing, timelike, with integrable orthogonal distribution) is complete [30, Thm. 2.2]. Note that a standard static spacetime $\left(I \times \Sigma,-\beta d t^{2} \oplus g_{\Sigma}\right)$ is globally hyperbolic if and only if the metric $\frac{1}{\beta} g_{\Sigma}$ is complete, in particular any standard static spacetime with closed $\Sigma$ is globally hyperbolic. We refer to the excellent survey [30] for further geometric and causal aspects of standard static spacetimes.

Thus, we shall consider in this section spacetimes that are conformally equivalent to standard static ones. Since we may first want a conformal characterisation of such spacetimes, we give the following

Proposition 3.2 A spacetime $\left(M^{n}, g\right)$ is conformally equivalent to a standard static spacetime if and only if there exists a smooth function $t: M \longrightarrow \mathbb{R}$ such that $\operatorname{grad}_{g}(t)$ is everywhere past-directed timelike and for the induced splitting $\left(M^{n}, g\right)=\left(I \times \Sigma,-\beta d t^{2} \oplus g_{t}\right)$ via the flow of $\frac{\operatorname{grad}_{g}(t)}{\left.\operatorname{|grad}_{g}(t)\right|_{g} ^{2}}$, the Riemannian metric $\frac{1}{\beta} g_{t}$ on $\Sigma$ does not depend on $t$.

A smooth function $t: M \longrightarrow \mathbb{R}$ whose gradient is everywhere past-directed timelike is called temporal, see e.g. [21, Def. 3.48]; a temporal function is in particular a time function, i.e., it is monotonously increasing on any future-directed causal curve in $\left(M^{n}, g\right)$. Note that the vector field - and hence the induced flow $-\frac{\operatorname{grad}_{g}(t)}{\left.\operatorname{grad}_{g}(t)\right|_{g} ^{2}}$, the conditions $t$ be a temporal function and $\frac{\partial}{\partial t}\left(\frac{1}{\beta} g_{t}\right)=0$ all only depend on the conformal class of $g$.

Clearly, a spacetime $\left(M^{n}, g\right)$ that is conformally equivalent to a standard static one has a (futuredirected) timelike conformal Killing vector field, the converse being wrong in general (though a globally hyperbolic spacetime with complete timelike conformal Killing vector field is conformally equivalent to a so-called standard stationary spacetime [30, Prop. 3.3]). In particular, globally hyperbolic spacetimes with trivial or even discrete conformal group cannot be conformally equivalent to a standard static one.

For instance, any warped product spacetime $\left(M^{n}, g\right)=\left(I \times \Sigma,-d t^{2} \oplus b(t)^{2} g_{\Sigma}\right)$, where $b \in C^{\infty}\left(I, \mathbb{R}_{+}^{\times}\right)$, admits such a temporal function (fix $s_{0} \in I$ and set $t(s, x):=\int_{s_{0}}^{s} \frac{d \tau}{b(\tau)}$ ) and hence is conformally equivalent to a standard static spacetime. More concretely, if $\left(M^{n}, g\right)=(] \alpha_{-}, \alpha_{+}\left[\times \Sigma,-d t^{2} \oplus\right.$ $\left.b(t)^{2} g_{\Sigma}\right)$ for some $b \in C^{\infty}(] \alpha_{-}, \alpha_{+}\left[, \mathbb{R}_{+}^{\times}\right)$, then fixing $\left.t_{0} \in\right] \alpha_{-}, \alpha_{+}[$, the map

$$
\begin{aligned}
\Phi:] \alpha_{-}, \alpha_{+}[\times \Sigma & \longrightarrow] a_{-}, a_{+}[\times \Sigma \\
(t, x) & \longmapsto(\psi(t), x),
\end{aligned}
$$

where $a_{ \pm}:=\int_{t_{0}}^{\alpha_{ \pm}} \frac{d s}{b(s)}$ and $\psi(t):=\int_{t_{0}}^{t} \frac{d s}{b(s)}$, is a smooth diffeomorphism with $\Phi^{*}\left(-d t^{2} \oplus g_{\Sigma}\right)=$ $-b^{-2} d t^{2} \oplus g_{\Sigma}=b^{-2} g$. 


\subsection{Existence of solutions to the Yamabe problem}

The first and most natural ansatz to solve the Yamabe problem in a product spacetime consists in separating variables.

Proposition 3.3 Let $\left(M^{n}, g\right)=\left(I \times \Sigma,-d t^{2} \oplus g_{\Sigma}\right)$, where $I \subset \mathbb{R}$ is an open interval, $\left(\Sigma^{n-1}, g_{\Sigma}\right)$ is a closed Riemannian manifold and $n \geq 3$. Let $S_{\bar{g}} \in \mathbb{R}, y \in C^{\infty}\left(I, \mathbb{R}_{+}^{\times}\right)$and $u \in C^{\infty}\left(\Sigma, \mathbb{R}_{+}^{\times}\right)$be arbitrary. Then the function $\varphi \in C^{\infty}\left(M, \mathbb{R}_{+}^{\times}\right), \varphi(t, x):=y(t) \cdot u(x)$, solves [2] if and only if

i) either $y$ or $u$ is constant in case $S_{\bar{g}} \neq 0$; if $y$ is constant, then $u$ solves $L_{g_{\Sigma}} u=a_{n} S_{\bar{g}} y^{p-2} u^{p-1}$ where $p:=\frac{2 n}{n-2}$; if $u$ is constant, then $S_{g_{\Sigma}}$ is constant and $y$ solves $y^{\prime \prime}+a_{n} S_{g_{\Sigma}} y=a_{n} S_{\bar{g}} u^{p-2} y^{p-1}$.

ii) the functions $y$ and $u$ satisfy $y^{\prime \prime}+\mu_{1}\left(L_{g_{\Sigma}}\right) y=0$ and $L_{g_{\Sigma}} u=\mu_{1}\left(L_{g_{\Sigma}}\right) u$ respectively in case $S_{\bar{g}}=0$, where $\mu_{1}\left(L_{g_{\Sigma}}\right) \in \mathbb{R}$ is the smallest eigenvalue of $L_{g_{\Sigma}}$.

Proof: By (6), the Yamabe equation (2) reads $\frac{\partial^{2} \varphi}{\partial t^{2}}+L_{g_{\Sigma}} \varphi=a_{n} S_{\bar{g}} \varphi^{p-1}$. For $\varphi$ of the form $\varphi(t, x):=y(\bar{t}) \cdot u(x)$, this becomes $y^{\prime \prime} \cdot u+y \cdot L_{g_{\Sigma}} u=a_{n} S_{\bar{g}}(y \cdot u)^{p-1}$. Dividing out by $y \cdot u$, this identity is equivalent to

$$
\frac{y^{\prime \prime}}{y}+\frac{L_{g_{\Sigma}} u}{u}=a_{n} S_{\bar{g}}(y \cdot u)^{p-2} .
$$

In case $S_{\bar{g}} \neq 0$, the first $t$-derivative of that identity gives $\left(\frac{y^{\prime \prime}}{y}\right)^{\prime}=(p-2) a_{n} S_{\bar{g}} u^{p-2} y^{p-3} y^{\prime}$, whose l.h.s. hence does not depend on $x \in \Sigma$, so that either $y^{\prime}=0$ on $I$ or $u$ is constant on $\Sigma$. If $y$ is constant on $I$, then $u$ solves $y \cdot L_{g_{\Sigma}} u=a_{n} S_{\bar{g}}(y \cdot u)^{p-1}$, that is, $L_{g_{\Sigma}} u=a_{n} S_{\bar{g}} y^{p-2} u^{p-1}$. If $u$ is constant on $\Sigma$, then by the identity just above $S_{g_{\Sigma}}$ must be constant and $y$ solves the ODE $y^{\prime \prime}+a_{n} S_{g_{\Sigma}} y=a_{n} S_{\bar{g}} u^{p-2} y^{p-1}$. This proves $\left.i\right)$.

In case $S_{\bar{g}}=0$, we obtain after differentiating w.r.t. $t$ the existence of a constant $\lambda \in \mathbb{R}$ with $\frac{y^{\prime \prime}}{y}=\lambda$ and hence also $\frac{L_{g_{\Sigma}} u}{u}=-\lambda$. In particular, $-\lambda$ is an eigenvalue with associated eigenfunction $u$ for the elliptic self-adjoint linear operator $L_{g_{\Sigma}}$ on $\Sigma$; but since we require $u>0$, the eigenvalue $-\lambda$ can only be the smallest one $\mu_{1}\left(L_{g_{\Sigma}}\right)$ by Courant's nodal domain theorem. This shows $\left.i i\right)$ and concludes the proof.

We concentrate on the equation $L_{g_{\Sigma}} u=\lambda u^{p-1}$ on $\Sigma$, for which existence results are well-known, see e.g. [20, Sec. 4] or [2, Sec. 2.3]:

Theorem 3.4 (H. Yamabe [34]) For $n \geq 3$ let $\left(\Sigma^{n-1}, g_{\Sigma}\right)$ be any closed Riemannian manifold. As above, let $L_{g_{\Sigma}}: C^{\infty}(\Sigma, \mathbb{R}) \longrightarrow C^{\infty}(\Sigma, \mathbb{R})$ be defined by $L_{g_{\Sigma}} \varphi:=\Delta_{g_{\Sigma}} \varphi+a_{n} S_{g_{\Sigma}} \varphi$, where $a_{n}:=\frac{n-2}{4(n-1)}$ and $S_{g_{\Sigma}}$ is the scalar curvature of $\left(\Sigma, g_{\Sigma}\right)$. For $p \in[2, \infty[$ consider the functional

$$
H^{1,2}(\Sigma) \backslash\{0\} \stackrel{E}{\longrightarrow} \mathbb{R}, \quad E(f):=\frac{\int_{\Sigma} f L_{g_{\Sigma}} f d \sigma}{\|f\|_{L^{p}(\Sigma)}^{2}}
$$

where $d \sigma$ is the Riemannian density associated to $g_{\Sigma}$ on $\Sigma$. Then we have the following:

i) An $f \in H^{1,2}(\Sigma) \backslash\{0\}$ is a critical point of $E$ if and only if it satisfies $L_{g_{\Sigma}} f=\frac{E(f)}{\|f\|_{L^{p}(\Sigma)}^{p-2}} \cdot f^{p-1}$.

ii) If $p \in\left[2, p^{*}\left[\right.\right.$, where $\left.\left.p^{*}:=\frac{2(n-1)}{n-3} \in\right] 2, \infty\right]$, then there exists a minimizer of $E$ on $H^{1,2}(\Sigma) \backslash\{0\}$.

In particular, there exists a $\varphi \in C^{\infty}\left(\Sigma, \mathbb{R}_{+}^{\times}\right)$with (w.l.o.g.) $\|\varphi\|_{L^{p}(\Sigma)}=1$ satisfying $L_{g_{\Sigma}} \varphi=$ $\lambda_{p}\left(\Sigma, g_{\Sigma}\right) \cdot \varphi^{p-1}$ on $\Sigma$, where $\lambda_{p}\left(\Sigma, g_{\Sigma}\right):=\inf _{H^{1,2}(\Sigma) \backslash\{0\}}(E) \in \mathbb{R}$. 
The sign of $\lambda_{p}\left(\Sigma, g_{\Sigma}\right)$ turns out to be that of the smallest eigenvalue of the elliptic self-adjoint operator $L_{g_{\Sigma}}$ :

Lemma 3.5 With the notations of Theorem 3.4 and $p \in\left[2, p^{*}\left[\right.\right.$, the constant $\lambda_{p}\left(\Sigma, g_{\Sigma}\right)$ and the smallest eigenvalue $\mu_{1}$ of $L_{g_{\Sigma}}$ have the same sign: the one is positive (resp. 0, negative) if and only if the other is positive (resp. 0, negative).

Proof: The negative case is clear: by definition of the constant $\lambda_{p}\left(\Sigma, g_{\Sigma}\right)$, it is negative if and only if there exists an $f \in H^{1,2}(\Sigma) \backslash\{0\}$ with $\int_{\Sigma} f L_{g_{\Sigma}} f d \sigma<0$, which, by the min-max principle, is equivalent to $\mu_{1}<0$. Now the condition $p \geq 2$ provides a trivial inequality between $\lambda_{p}\left(\Sigma, g_{\Sigma}\right)$ and $\mu_{1}$ : since $\Sigma$ is closed, we have, using Hölder's inequality, $\|\cdot\|_{2} \leq C \cdot\|\cdot\|_{p}$ for some constant $C=C\left(\Sigma, g_{\Sigma}\right)$, hence

$$
\frac{\int_{\Sigma} f L_{g_{\Sigma}} f d \sigma}{\|f\|_{2}^{2}} \geq C^{\prime} \cdot \frac{\int_{\Sigma} f L_{g_{\Sigma}} f d \sigma}{\|f\|_{p}^{2}} \geq C^{\prime} \cdot \lambda_{p}\left(\Sigma, g_{\Sigma}\right)
$$

for some constant $C^{\prime}=C^{\prime}\left(\Sigma, g_{\Sigma}\right)$ and for every $f \in H^{1,2}(\Sigma) \backslash\{0\}$; the min-max principle yields $\mu_{1} \geq C^{\prime} \cdot \lambda_{p}\left(\Sigma, g_{\Sigma}\right)$. So, if $\lambda_{p}\left(\Sigma, g_{\Sigma}\right)=0$, then this inequality implies $\mu_{1} \geq 0$; on the other hand, Theorem 3.4 provides the existence of an $f \in C^{\infty}\left(\Sigma, \mathbb{R}_{+}^{\times}\right)$with $L_{g_{\Sigma}} f=0$, in particular 0 is an eigenvalue of $L_{g_{\Sigma}}$ and hence $\mu_{1} \leq 0$, so $\mu_{1}=0$. Conversely, if $\mu_{1}=0$, then the above inequality provides $\lambda_{p}\left(\Sigma, g_{\Sigma}\right) \leq 0$; on the other hand, $\int_{\Sigma} f L_{g_{\Sigma}} f d \sigma \geq 0$ holds by the min-max principle, so that $\lambda_{p}\left(\Sigma, g_{\Sigma}\right) \geq 0$ and therefore $\lambda_{p}\left(\Sigma, g_{\Sigma}\right)=0$. This concludes the proof.

For instance, if $S_{g_{\Sigma}}=0$, then it is clear that $\mu_{1}=\lambda_{p}\left(\Sigma, g_{\Sigma}\right)=0$ (take $\varphi$ to be constant on $\Sigma$ ). If $S_{g_{\Sigma}}>0$ on $\Sigma$, then $\lambda_{p}\left(\Sigma, g_{\Sigma}\right)>0$, as one can deduce from the bounded Sobolev embedding $H^{1,2}(\Sigma) \hookrightarrow L^{p}(\Sigma)$ (recall that $\left.p \leq \frac{2(n-1)}{n-3}\right)$ : there exists a constant $C=C\left(\Sigma, g_{\Sigma}\right)>0$ such that, for every $f \in H^{1,2}(\Sigma) \backslash\{0\}$,

$$
\int_{\Sigma} f L_{g_{\Sigma}} f d \sigma \geq \min \left(1, a_{n} \min _{\Sigma}\left(S_{g_{\Sigma}}\right)\right) \cdot \underbrace{\int_{\Sigma}|d f|^{2}+f^{2} d \sigma}_{\|f\|_{H^{1,2}(\Sigma)}} \geq C \cdot \min \left(1, a_{n} \min _{\Sigma}\left(S_{g_{\Sigma}}\right)\right) \cdot\|f\|_{p}^{2},
$$

from which we deduce $\lambda_{p}\left(\Sigma, g_{\Sigma}\right) \geq C \cdot \min \left(1, a_{n} \min _{\Sigma}\left(S_{g_{\Sigma}}\right)\right)$. In particular, $\lambda_{p}\left(\Sigma, g_{\Sigma}\right)>0$ as soon as $\min _{\Sigma}\left(S_{g_{\Sigma}}\right)>0$. More generally, if $S_{g_{\Sigma}} \geq 0$ and does not identically vanish on $\Sigma$, then $\int_{\Sigma} u_{1}\left(L_{g_{\Sigma}} u_{1}\right) d \sigma>0$ for any (non-zero) eigenfunction $u_{1}$ associated to the smallest eigenvalue $\mu_{1}$, in particular $\mu_{1}>0$ and hence $\lambda_{p}\left(\Sigma, g_{\Sigma}\right)>0$. Note that, if $\mu_{1}<0$ - or, equivalently, $\lambda_{p}\left(\Sigma, g_{\Sigma}\right)<0$ - implies $\min _{\Sigma}\left(S_{g_{\Sigma}}\right)<0$, however the other implication is wrong (use e.g. a continuity argument: perturb appropriately the standard metric on $\mathbb{S}^{n}$ so as to make the scalar curvature negative somewhere while keeping $\lambda_{p}$ positive). Beware also that $\lambda_{p}\left(\Sigma, g_{\Sigma}\right)$ is not a conformal invariant - it is in particular not the infimum of the standard Yamabe functional.

The first global existence result of that section is the following

Theorem 3.6 Let a spacetime $\left(M^{n}, g\right)$ be conformally equivalent to the Lorentzian product $(I \times$ $\left.\Sigma,-d t^{2} \oplus g_{\Sigma}\right)$ of an open interval $I \subset \mathbb{R}$ with a closed Riemannian manifold $\Sigma^{n-1}$, where $n \geq 3$. Let $\lambda_{p}\left(\Sigma, g_{\Sigma}\right):=\inf _{H^{1,2}(\Sigma) \backslash\{0\}}(E) \in \mathbb{R}$ (see Theorem 3.4) and $p:=\frac{2 n}{n-2}$. Then for $S_{\bar{g}}:=\frac{\lambda_{p}\left(\Sigma, g_{\Sigma}\right)}{a_{n}}$ there exists a $\varphi \in C^{\infty}\left(M, \mathbb{R}_{+}^{\times}\right)$solving $[2]$.

Proof: By conformal invariance of the Yamabe problem, we may assume that $\left(M^{n}, g\right)=(I \times$ $\left.\Sigma,-d t^{2} \oplus g_{\Sigma}\right)$. In that case, 22 becomes $\frac{\partial^{2} \varphi}{\partial t^{2}}+L_{g_{\Sigma}} \varphi=a_{n} S_{\bar{g}} \varphi^{p-1}$ by Lemma 2.3 Since 
$p \in\left[2, \frac{2(n-1)}{n-3}[\right.$, Theorem 3.4 provides the existence of a smooth positive solution $\varphi$ on $\Sigma$ of $L_{g_{\Sigma}} \varphi=\lambda_{p}\left(\Sigma, g_{\Sigma}\right) \cdot \varphi^{p-1}$. This $\varphi$ does not depend on $t$, hence solves $(2)$.

As a consequence, every warped product spacetime admits at least one solution to the Yamabe problem.

\section{Notes 3.7}

1. The proof of Theorem 3.6 actually shows that the same statement as in Theorem 3.6 holds true for any (necessarily non globally hyperbolic) spacetime conformally equivalent to $\left(\mathbb{S}^{1} \times\right.$ $\left.\Sigma^{n-1},-d t^{2} \oplus g_{\Sigma}\right)$ with closed $\Sigma$, where $\mathbb{S}^{1}$ is a circle of arbitrary length: the solution we construct does not depend on time and is therefore periodic.

2. One need not have uniqueness (up to scaling by a positive constant) of a conformal metric with constant scalar curvature. Take e.g. $\left(M^{n}, g\right):=\left(\mathbb{R} \times \mathbb{T}^{n-1},-d t^{2} \oplus\right.$ can $)$, where $\mathbb{T}^{n-1}=$ $\mathbb{R}^{n-1} / \mathbb{Z}^{n-1}$ is the $n-1$-dimensional torus obtained by modding out $\mathbb{R}^{n-1}$ by the canonically embedded lattice $\mathbb{Z}^{n-1} \subset \mathbb{R}^{n-1}$ and can is the induced flat metric on $\mathbb{T}^{n-1}$. Taking any two 1-periodic functions $v, w \in C^{\infty}\left(\mathbb{R}, \mathbb{R}_{+}^{\times}\right)$, the function $\varphi \in C^{\infty}\left(\mathbb{R} \times \mathbb{R}^{n-1}, \mathbb{R}_{+}^{\times}\right)$defined by

$$
\varphi(t, x):=v\left(t+x_{1}\right)+w\left(t-x_{1}\right)
$$

for all $t \in \mathbb{R}$ and $x=\left(x_{1}, \ldots, x_{n-1}\right) \in \mathbb{R}^{n-1}$, satisfies $\square \varphi=0$ and induces a smooth function (also denoted by $\varphi$ ) on $\mathbb{R} \times \mathbb{T}^{n-1}$ satisfying the same equation. Therefore, one obtains a whole family of non-trivial conformal metrics with vanishing scalar curvature on $M^{n}$. This also shows a big difference with the Riemannian setting, where every conformal metric with vanishing scalar curvature on $\mathbb{R} \times \mathbb{T}^{n-1}$ must be a constant positive multiple of the metric $d t^{2} \oplus$ can by Liouville's theorem (implying that every positive harmonic function on $\mathbb{R}^{n}$ must be constant). Uniqueness of the solutions is further discussed in Section 3.2 below.

Theorem 3.6 shows the existence of at least one conformal metric with constant scalar curvature on any conformally standard static spacetime. However, we notice that the sign of that conformal scalar curvature is given by that of the conformal invariant $\lambda_{p}\left(\Sigma, g_{\Sigma}\right)$ defined in Theorem 3.4 Therefore, we are led to asking whether any constant scalar curvature may be prescribed in any conformal class, and if not, how "large" the maximal domain of existence for solutions is. For this, the following lemma is useful.

Lemma 3.8 (Grönwall) Let $\alpha, \beta: I \longrightarrow \mathbb{R}$ be continuous functions and $t_{0} \in I$ be arbitrary.

1) If $y^{\prime}+\alpha(t) y \leq 0$, then $y(t)-y\left(t_{0}\right) e^{-\int_{t_{0}}^{t} \alpha(s) d s}\left\{\begin{array}{ll}\leq 0 & \text { if } t \geq t_{0} \\ \geq 0 & \text { if } t \leq t_{0}\end{array}\right.$.

2) If $y^{\prime \prime}+\alpha(t) y^{\prime}+\beta(t) y \leq 0$, then $y(t) \leq y\left(t_{0}\right) y_{0}+y^{\prime}\left(t_{0}\right) z_{0}$ for every $t \in I$, where $y_{0}, z_{0}$ solve the differential equation $w^{\prime \prime}+\alpha(t) w^{\prime}+\beta(t) w=0$ on I with initial conditions $y_{0}\left(t_{0}\right)=1=z_{0}^{\prime}\left(t_{0}\right)$ and $y_{0}^{\prime}\left(t_{0}\right)=0=z_{0}\left(t_{0}\right)$. In other words, $y$ must be lower than or equal to the solution of the corresponding differential equation with the same initial conditions at $t_{0}$.

We come to the main existence result of this section.

Theorem 3.9 Let a spacetime $\left(M^{n}, g\right)$ be conformally equivalent to the Lorentzian product $(I \times$ $\left.\Sigma,-d t^{2} \oplus g_{\Sigma}\right)$ of an open interval $I \subset \mathbb{R}$ with a closed Riemannian manifold $\left(\Sigma^{n-1}, g_{\Sigma}\right)$, where $n \geq 3$. Let $\mu_{1}\left(L_{g_{\Sigma}}\right) \in \mathbb{R}$ be the smallest eigenvalue of $L_{g_{\Sigma}}$ and let $S_{\bar{g}} \in \mathbb{R}$ be an arbitrary constant.

1) If $\mu_{1}\left(L_{g_{\Sigma}}\right)<0$, then 
1a) either $S_{\bar{g}} \leq 0$ and then (2) has a globally defined smooth positive solution on $M^{n}$,

1b) or $S_{\bar{g}}>0$ and then (2) has no globally defined smooth positive solution on $M^{n}=I \times \Sigma$ if $I=\mathbb{R}$.

2) If $\mu_{1}\left(L_{g_{\Sigma}}\right)=0$, then

2a) either $S_{\bar{g}}<0$ and then (2) has no globally defined smooth positive solution on $M^{n}=I \times \Sigma$ if $I=\mathbb{R}$.

2b) or $S_{\bar{g}}=0$ and then (2) has a globally defined smooth positive solution on $M^{n}$,

2c) or $S_{\bar{g}}>0$ and then (2) has no globally defined smooth positive solution on $M^{n}=I \times \Sigma$ if $I=\mathbb{R}$.

3) If $\mu_{1}\left(L_{g_{\Sigma}}\right)>0$, then

$3 a$ ) either $S_{\bar{g}}<0$ and then (2) has a globally defined smooth positive solution on $M^{n}=I \times \Sigma$ only if $|I| \leq \frac{\pi}{\sqrt{\mu_{1}\left(L_{g_{\Sigma}}\right)}}$,

3b) or $S_{\bar{g}}=0$ and then (2) has a globally defined smooth positive solution on $M^{n}=I \times \Sigma$ if and only if $|I| \leq \frac{\pi}{\sqrt{\mu_{1}\left(L_{g_{\Sigma}}\right)}}$,

3c) or $S_{\bar{g}}>0$ and then (2) has a globally defined smooth positive solution on $M^{n}$.

Proof of Theorem 3.9. Note that the statements $1 a$ ) for the subcase $S_{\bar{g}}<0,2 b$ ) and $3 c$ ) are already contained in Theorem 3.6 via Lemma 3.5 and after possibly rescaling the solution so as to adjust the constant on the r.h.s.

We show how to obtain in all cases a necessary condition for the existence of a global solution to 22. Given any constant $S_{\bar{g}} \in \mathbb{R}$, assume $\varphi \in C^{\infty}\left(M, \mathbb{R}_{+}^{\times}\right)$is a solution to (2). Again, we may assume that $(M, g)=\left(I \times \Sigma,-d t^{2} \oplus g_{\Sigma}\right)$. Let $u$ be any positive (necessarily smooth) eigenfunction associated to the smallest eigenvalue $\mu_{1}\left(L_{g_{\Sigma}}\right)$ of $L_{g_{\Sigma}}$. Multiplying (2) with $u$ and integrating w.r.t. the Riemannian measure $d \sigma$ associated to $g_{\Sigma}$ on $\Sigma$, we obtain, using the formal self-adjointness of $L_{g_{\Sigma}}$ :

$$
\begin{aligned}
a_{n} S_{\bar{g}} \int_{\Sigma} \varphi^{p-1}(t, x) u(x) d \sigma(x) & \stackrel{2}{=} \int_{\Sigma}\left(\square \varphi+a_{n} S_{g} \varphi\right)(t, x) u(x) d \sigma(x) \\
& \stackrel{6}{6} \int_{\Sigma}\left\{\frac{\partial^{2} \varphi}{\partial t^{2}}(t, x) u(x)+\left(L_{g_{\Sigma}} \varphi\right)(t, x) u(x)\right\} d \sigma(x) \\
& =\frac{d^{2}}{d t^{2}}\left(\int_{\Sigma} \varphi(t, \cdot) u d \sigma\right)+\int_{\Sigma} \varphi(t, \cdot) L_{g_{\Sigma}} u d \sigma \\
& =\frac{d^{2}}{d t^{2}}\left(\int_{\Sigma} \varphi(t, \cdot) u d \sigma\right)+\mu_{1}\left(L_{g_{\Sigma}}\right) \int_{\Sigma} \varphi(t, \cdot) u d \sigma
\end{aligned}
$$

where $p=\frac{2 n}{n-2}$. Therefore, the smooth positive function $y: I \rightarrow \mathbb{R}_{+}^{\times}, t \mapsto \int_{\Sigma} \varphi(t, \cdot) u d \sigma$, satisfies

$$
y^{\prime \prime}+\mu_{1}\left(L_{g_{\Sigma}}\right) y=a_{n} S_{\bar{g}} \int_{\Sigma} \varphi^{p-1}(t, \cdot) u d \sigma
$$

on $I$. An immediate consequence of this is that, if $S_{\bar{g}}=0$, then the existence of a smooth positive solution to (2) is actually equivalent to that of a smooth positive solution to (8): it is necessary by the above argument and, conversely, if some $y \in C^{\infty}\left(I, \mathbb{R}_{+}^{\times}\right)$solves $(8)$, then Proposition 3.3 implies that, for any positive (smooth) eigenfunction $u$ associated to the smallest eigenvalue $\mu_{1}\left(L_{g_{\Sigma}}\right)$ of $L_{g_{\Sigma}}$, the function $\varphi(t, x):=y(t) \cdot u(x)>0$ solves $\square \varphi+a_{n} S_{g} \varphi=0$ on $M$. Since obviously a 
positive smooth solution to the ODE (8) with $S_{\bar{g}}=0$ exists for $\mu_{1}\left(L_{g_{\Sigma}}\right) \leq 0$, we obtain $\left.1 a\right)$ for the subcase $S_{\bar{g}}=0$ (as well as $\left.2 b\right)$ ). For $\mu_{1}\left(L_{g_{\Sigma}}\right)>0$, any solution to 8 with $S_{\bar{g}}=0$ is of the form $t \mapsto A \cos \left(\sqrt{\mu_{1}\left(L_{g_{\Sigma}}\right)} t+c\right), A, c \in \mathbb{R}$, so that the existence of (at least) a positive solution (8) is equivalent to the length of $I$ being no greater than the half of the period of $t \mapsto \cos \left(\sqrt{\mu_{1}\left(L_{g_{\Sigma}}\right)} t\right)$, i.e., to $|I| \leq \frac{\pi}{\sqrt{\mu_{1}\left(L_{g_{\Sigma}}\right)}}$. This proves $\left.3 b\right)$.

Assume now $S_{\bar{g}}<0$ and $\mu_{1}\left(L_{g_{\Sigma}}\right) \geq 0$. If $\varphi \in C^{\infty}\left(M, \mathbb{R}_{+}^{\times}\right)$solves $(2)$, then by 8 the function $y$ defined as above from $\varphi$ satisfies $y^{\prime \prime}+\mu_{1}\left(L_{g_{\Sigma}}\right) y<0$ on $I$. If $\mu_{1}\left(L_{g_{\Sigma}}\right)=0$, then $y^{\prime \prime}<0$ on $I$, so that $y$ is strictly concave and hence has to change sign if $I=\mathbb{R}$. This shows $2 a)$. If $\mu_{1}\left(L_{g_{\Sigma}}\right)>0$, then fix any $t_{0} \in I$. By Lemma 3.8 the function $y$ must satisfy $y \leq z$, where $z \in C^{\infty}(I, \mathbb{R})$ solves $z^{\prime \prime}+\mu_{1}\left(L_{g_{\Sigma}}\right) z=0$ on $I$ with $z\left(t_{0}\right)=y\left(t_{0}\right)$ as well as $z^{\prime}\left(t_{0}\right)=y^{\prime}\left(t_{0}\right)$. Since $z-$ and hence also $y-$ can remain positive only on an interval of length at most $\frac{\pi}{\sqrt{\mu_{1}\left(L_{g_{\Sigma}}\right)}}$ (see just above), the length $|I|$ of $I$ must satisfy $|I| \leq \frac{\pi}{\sqrt{\mu_{1}\left(L_{g_{\Sigma}}\right)}}$. This shows $\left.3 a\right)$.

In the remaining case where $S_{\bar{g}}>0$ and $\mu_{1}\left(L_{g_{\Sigma}}\right) \leq 0$, the identity (8) implies that, if $\varphi \in$ $C^{\infty}\left(M, \mathbb{R}_{+}^{\times}\right)$solves $(2)$, then for any smooth positive $u \in \operatorname{Ker}\left(L_{g_{\Sigma}}-\mu_{1}\left(L_{g_{\Sigma}}\right)\right)$, the smooth positive function $y(t):=\int_{\Sigma} \varphi(t, \cdot) u d \sigma$ satisfies

$$
y^{\prime \prime} \geq y^{\prime \prime}+\mu_{1}\left(L_{g_{\Sigma}}\right) y=a_{n} S_{\bar{g}} \int_{\Sigma}\left(\varphi(t, \cdot) u^{\frac{1}{p-1}}\right)^{p-1} d \sigma
$$

on $I$. But since $u$ is continuous and positive on the compact space $\Sigma$, there is a positive constant $C$ (depending on $p=\frac{2 n}{n-2}$ and $u$ ) such that $u^{\frac{1}{p-1}} \geq C u$, so that, by Hölder inequality,

$$
y^{\prime \prime} \geq a_{n} S_{\bar{g}} C^{p-1} \int_{\Sigma}(\varphi(t, \cdot) u)^{p-1} d \sigma \geq \frac{a_{n} S_{\bar{g}} C^{p-1}}{\operatorname{Vol}\left(\Sigma, g_{\Sigma}\right)^{p-2}} y^{p-1}
$$

on $I$. This leads to an explosion of $y$ in finite time and hence to a contradiction in case $I=\mathbb{R}$. Namely we may first assume, up to changing $y$ into $t \mapsto y(\alpha t)$ for some $\alpha>0$, that

$$
y^{\prime \prime} \geq \frac{p}{2} y^{p-1}
$$

on $\mathbb{R}$. Since then $y$ is strictly convex, only two (non disjoint) situations can occur: there is an interval of the form $\left[t_{0}, \infty\left[\right.\right.$ on which $y^{\prime} \geq 0$ or there is an interval of the form $\left.]-\infty, t_{0}\right]$ on which $y^{\prime} \leq 0$. In the latter case, up to changing $t$ into $-t$ - which does not modify $(9)$ - we can again assume that $y^{\prime} \geq 0$ on some interval of the form $\left[t_{0}, \infty\left[\right.\right.$. Up to translating by $t_{0}$, we can also assume that $t_{0}=0$. Since $y^{\prime} \geq 0$ on $\left[0, \infty\left[\right.\right.$, the identity $\sqrt{9}$ yields $2 y^{\prime \prime} y^{\prime} \geq p y^{p-1} y^{\prime}$ on $[0, \infty[$, hence $\left(y^{\prime}\right)^{2}(t)-\left(y^{\prime}\right)^{2}(0) \geq y^{p}(t)-y^{p}(0)$ for any $t \geq 0$, in particular $y^{\prime} \geq \sqrt{y^{p}-y^{p}(0)}$ on $[0, \infty[$. The latter inequality gives

$$
\int_{y(0)}^{y(t)} \frac{d z}{\sqrt{z^{p}-y^{p}(0)}} \geq t
$$

for any $t \geq 0$. Now since $p>2$ the integral $\int_{y(0)}^{\infty} \frac{d z}{\sqrt{z^{p}-y^{p}(0)}}$ converges, that is, the domain where $y(t)$ is defined is bounded above, or, equivalently, $y(t) \rightarrow \infty$ as $t \rightarrow T$ for some $T<\infty$. This shows $1 b$ ) and $2 c$ ) and concludes the proof of Theorem 3.9

Note that, in the cases $1 b), 2 a), 2 c$ ) and $3 a$ ), local existence of solutions to (2) implies anyway the existence of a smooth positive solution $\varphi$ to (2) on $I \times \Sigma$ for sufficiently short $|I|$. Even if it looks like it, global existence of solutions has nothing to do with timelike geodesic completeness of the product metric (which is anyway not a conformal invariant), see de Sitter spacetime below. For further ODE-like obstructions to the existence of particular metrics in (pseudo-)Riemannian 
conformal classes, we refer to [23].

A first application of Theorem 3.9 is the following surprising example, where we see there exist spacetimes with positive scalar curvature admitting conformal metrics with vanishing scalar curvature - and this only in low dimensions.

Corollary 3.10 Let a spacetime $\left(M^{n}, g\right)$ be conformally equivalent to the warped product $(\mathbb{R} \times$ $\left.\Sigma^{n-1},-d t^{2} \oplus \cosh (t)^{2} g_{\Sigma}\right)$ of $\mathbb{R}$ with a closed Riemannian manifold $\left(\Sigma^{n-1}, g_{\Sigma}\right)$ of constant scalar curvature $(n-1)(n-2)$ and with warping function $b=\cosh$. Then there exists a conformal metric with vanishing scalar curvature on $\left(M^{n}, g\right)$ if and only if $n \leq 4$.

Proof: Note that, by (5), the scalar curvature of $\left(M^{n}, g\right)$ is $S_{g}=n(n-1)>0$. We have already constructed an explicit isometry between $\left(M^{n}, b^{-2} g\right.$ ) (which is conformally equivalent to $\left(M^{n}, g\right)$ ) and (]$a_{-}, a_{+}\left[\times \Sigma^{n-1},-d t^{2} \oplus g_{\Sigma}\right)$, where $b(t):=\cosh (t)$ and $a_{ \pm}:=\int_{0}^{ \pm \infty} \frac{d s}{b(s)}$ : set $\Phi(t, x):=(\psi(t), x)$ with $\psi(t):=\int_{0}^{t} \frac{d s}{b(s)}$. It is elementary to compute $\psi(t)=2 \int_{0}^{t} \frac{e^{-s} d s}{1+e^{-2 s}}=\frac{\pi}{2}-2 \arctan \left(e^{-t}\right)$, so that $a_{ \pm}= \pm \frac{\pi}{2}$. Now since $S_{g_{\Sigma}}=(n-1)(n-2)$ is constant, $\mu_{1}\left(L_{g_{\Sigma}}\right)=a_{n} S_{g_{\Sigma}}=\frac{(n-2)^{2}}{4}$, so that, by Theorem 3.9 there exists a positive solution to 2 with $S_{\bar{g}}=0$ if and only if $a_{+}-a_{-} \leq \frac{\pi}{\sqrt{\mu_{1}}}$, that is, if and only if $\pi \leq \frac{2 \pi}{n-2}$, that is, if and only if $n \leq 4$.

For instance, if $\left(M^{n}, g\right):=\left(\mathbb{R} \times \mathbb{S}^{n-1},-d t^{2} \oplus \cosh (t)^{2}\right.$ can $)$ is the de Sitter spacetime of constant sectional curvature 1 , where $\left(\mathbb{S}^{n-1}\right.$, can) is the round sphere (of constant sectional curvature 1 if $n \geq 3$ ), then Corollary 3.10 shows that the existence of a conformal metric with vanishing scalar curvature is equivalent to $n \leq 4$.

Note 3.11 There is something deeply unsatisfying about Theorem 3.9 although the results we obtain are by nature conformally invariant, the assumptions we work with are not. For recall that we have first chosen a foliation by spacelike hypersurfaces - or, equivalently, a temporal function on the spacetime. Even more disturbing is the fact that the sign of the first eigenvalue of the Laplacetype operator $L_{g_{\Sigma}}$ on each leaf $\Sigma$ can change when fixing the foliation but changing the metric conformally on the spacetime. This remark is crucial when wanting to generalise the existence results to arbitrary globally hyperbolic spacetimes.

\subsection{Uniqueness of solutions to the Yamabe problem}

Next we turn to the uniqueness issue for the Yamabe problem. As we already noticed, given a globally hyperbolic spacetime $M^{n}$ with closed spacelike Cauchy hypersurface $\Sigma$ having future unit normal $\nu$, the local well-posedness of the Cauchy problem $\square \varphi+a_{n} S_{g} \varphi=a_{n} S_{\bar{g}} \varphi^{\frac{n+2}{n-2}}$ on $M, \varphi_{\left.\right|_{\Sigma}}=\varphi_{0}$ and $\partial_{\nu} \varphi=\varphi_{1}$ on $\Sigma$, ensures - at least in a neighbourhood of $\Sigma$ - the existence of infinitely many "independent" local solutions to the Yamabe problem. Therefore the only interesting question in this respect deals with the global aspects of uniqueness.

We start with looking at the ODE $y^{\prime \prime}+a_{n} S_{g_{\Sigma}} y=a_{n} S_{\bar{g}} y^{p-1}$ from Proposition 3.3 on $I \subset \mathbb{R}$ and under the assumption that the scalar curvature $S_{g_{\Sigma}}$ of $\left(\Sigma, g_{\Sigma}\right)$ is constant. It is easy to see what happens for $S_{\bar{g}}=0$ : if $S_{g_{\Sigma}}<0$, then there always exists a 2-parameter-family of positive solutions to $y^{\prime \prime}+a_{n} S_{g_{\Sigma}} y=0$ on $I$; if $S_{g_{\Sigma}}=0$, then only constant solutions $y>0$ to $y^{\prime \prime}=0$ can remain positive on $\mathbb{R}$; in case $S_{g_{\Sigma}}>0$, there is no positive solution to $y^{\prime \prime}+a_{n} S_{g_{\Sigma}} y=0$ on $\mathbb{R}$ (but obviously there is one and even a 2-parameter-family of solutions on a sufficiently small interval). In the case $S_{\bar{g}} \neq 0$, we may assume, up to multiplying $y$ by a positive constant, that $a_{n} S_{\bar{g}}=\varepsilon \frac{p}{2}$ with $\varepsilon \in\{ \pm 1\}$. 
Lemma 3.12 Given $\left.s \in \mathbb{R}_{+}^{\times}, p \in\right] 2, \infty\left[\right.$ and $\varepsilon \in\{ \pm 1\}$, consider the $O D E y^{\prime \prime}=\varepsilon\left(\frac{p}{2} y^{p-1}-s y\right)$ on some open interval $I \subset \mathbb{R}$.

1) If $\varepsilon=1$, then the only positive solution to that $O D E$ on $\mathbb{R}$ is the constant one $y=\left(\frac{2 s}{p}\right)^{\frac{1}{p-2}}$.

2) If $\varepsilon=-1$, then there are infinitely many non-constant positive solutions to that ODE on I. More precisely, for any $T \in] \frac{2 \pi}{\sqrt{(p-2) s}}, \infty[$, there exists a $T$-periodic positive solution to $y^{\prime \prime}=-\frac{p}{2} y^{p-1}+$ sy on $\mathbb{R}$.

Proof: If $y$ solves $y^{\prime \prime}=\varepsilon\left(\frac{p}{2} y^{p-1}-s y\right)$, then multiplying with $y^{\prime}$ and integrating one obtains

$$
\left(y^{\prime}\right)^{2}=\varepsilon F(y)-\lambda
$$

for some $\lambda \in \mathbb{R}$, where $F: \mathbb{R}_{+} \rightarrow \mathbb{R}, F(y):=y^{p}-s y^{2}$. Therefore, we just have to investigate the qualitative behaviour of solutions to the first-order ODE $\left(y^{\prime}\right)^{2}=\varepsilon F(y)-\lambda$ according to the value of $\lambda$. This equation can be solved in the form $t=t(y)= \pm \int^{y} \frac{d z}{\sqrt{\varepsilon F(z)-\lambda}}$ according to the sign of $y^{\prime}$ on the interval under consideration. Moreover, any solution to $\left(y^{\prime}\right)^{2}=\varepsilon F(y)-\lambda$ which is not a critical point of $F$ is a solution to the original equation $y^{\prime \prime}=\varepsilon\left(\frac{p}{2} y^{p-1}-s y\right)$. Hence we first have to determine the regular and critical values of $F$. A short computation gives the two critical values 0 and $-\frac{p-2}{p}\left(\frac{2 s}{p}\right)^{\frac{p}{p-2}}$ for $F$, with corresponding critical points 0 and $\left(\frac{2 s}{p}\right)^{\frac{1}{p-2}}$ respectively. We start with the case $\varepsilon=1$ :

- Any $\lambda \in \mathbb{R}_{+}^{\times}$is a regular value of $F$ and $F^{-1}(\{\lambda\})=\left\{x_{\lambda}\right\}$ with $\left.x_{\lambda} \in\right]^{\frac{1}{p^{-2}}}, \infty[$. Because $p>2$ we have $\int_{x_{\lambda}+1}^{\infty} \frac{d y}{\sqrt{F(y)-\lambda}}<\infty$, so that any solution $y$ corresponding to $\lambda>0$ explodes in finite time and therefore cannot exist on $\mathbb{R}$.

- For $\lambda=0$, apart from the trivial solution $y=0$ (we exclude anyway), the only solution shows exactly the same behaviour as before.

- For $\lambda \in]-\frac{p-2}{p}\left(\frac{2 s}{p}\right)^{\frac{p}{p-2}}, 0\left[\right.$, the preimage $F^{-1}\left(\left[\lambda, \infty[)\right.\right.$ consists of two intervals of the form $\left[0, x_{\lambda}^{-}\right]$ and $\left[x_{\lambda}^{+}, \infty\left[\right.\right.$ respectively, with $0<x_{\lambda}^{-}<\left(\frac{2 s}{p}\right)^{\frac{1}{p-2}}<x_{\lambda}^{+}<s^{\frac{1}{p-2}}$. Since $\lambda$ is a regular value of $F$, the behaviour of the solution taking its values in $\left[x_{\lambda}^{+}, \infty[\right.$ is the same as before (explosion in finite time); for $\left[0, x_{\lambda}^{-}\right]$the solution vanishes in finite time because of $\int_{0}^{\frac{x_{\lambda}^{-}}{2}} \frac{d y}{\sqrt{F(y)-\lambda}}<\infty$. In both cases, $y$ is not everywhere positive or is not defined on $\mathbb{R}$.

- For $\lambda=-\frac{p-2}{p}\left(\frac{2 s}{p}\right)^{\frac{p}{p-2}}$, apart from the constant solution $\left(\frac{2 s}{p}\right)^{\frac{1}{p-2}}$, we have two kinds of behaviour for $y$ according to one value $y\left(t_{0}\right)$ of $y$ lying in $] 0,\left(\frac{2 s}{p}\right)^{\frac{1}{p-2}}$ [ or in $]\left(\frac{2 s}{p}\right)^{\frac{1}{p-2}}, \infty[$. If $\left.y\left(t_{0}\right) \in\right]\left(\frac{2 s}{p}\right)^{\frac{1}{p-2}}, \infty[$, then $y$ explodes in finite time on one side and attains the critical point $\left(\frac{2 s}{p}\right)^{\frac{1}{p-2}}$ in infinite time on the other. If $\left.y\left(t_{0}\right) \in\right] 0,\left(\frac{2 s}{p}\right)^{\frac{1}{p-2}}$ [, then $y$ vanishes in finite time on the one side and attains the critical point $\left(\frac{2 s}{p}\right)^{\frac{1}{p-2}}$ in infinite time on the other. Again, no non-constant positive solution is defined on $\mathbb{R}$.

- For $\lambda \in]-\infty,-\frac{p-2}{p}\left(\frac{2 s}{p}\right)^{\frac{p}{p-2}}$ [ the function $y^{\prime}$ cannot change sign; the solution $y$ must vanish in finite time on the one side and explode in finite time on the other.

This shows 1). The case $\varepsilon=-1$ can also be divided in different subcases, compare [31, pp. 132-135]:

- For $\lambda \in] \frac{p-2}{p}\left(\frac{2 s}{p}\right)^{\frac{p}{p-2}}, \infty\left[\right.$, there is of course no solution to $\left(y^{\prime}\right)^{2}=-F(y)-\lambda$. 
- For $\lambda=\frac{p-2}{p}\left(\frac{2 s}{p}\right)^{\frac{p}{p-2}}$, the only solution to $\left(y^{\prime}\right)^{2}=-F(y)-\lambda$ is the constant one $y=\left(\frac{2 s}{p}\right)^{\frac{1}{p-2}}$.

- For $\lambda \in] 0, \frac{p-2}{p}\left(\frac{2 s}{p}\right)^{\frac{p}{p-2}}\left[\right.$, the preimage $(-F)^{-1}\left(\left[\lambda, \infty[)=\left[x_{\lambda}^{-}, x_{\lambda}^{+}\right]\right.\right.$, where $0<x_{\lambda}^{-}<\left(\frac{2 s}{p}\right)^{\frac{1}{p-2}}<$ $x_{\lambda}^{+}<s^{\frac{1}{p-2}}$. This time, $y$ is periodic (in particular defined on $\mathbb{R}$ ) and oscillates between the values $x_{\lambda}^{-}$and $x_{\lambda}^{+}$. Its period $T_{\lambda}$ (depending on $\lambda$ ) is given by $T_{\lambda}=2 \int_{x_{\lambda}^{-}}^{x_{\lambda}^{+}} \frac{d y}{\sqrt{-F(y)-\lambda}}$, which can be easily seen to depend continuously on $\lambda$ (since $x_{\lambda}^{ \pm}$do) with $T_{\lambda} \underset{\lambda \rightarrow 0^{+}}{\longrightarrow} \infty$ as well as $T_{\lambda} \underset{\lambda \rightarrow \frac{p-2}{p}\left(\frac{2 s}{p}\right)^{\frac{p}{p-2}}-}{\longrightarrow} \frac{2 \pi}{\sqrt{(p-2) s}}>0$, which is the period for the linearized equation $y^{\prime \prime}=-(p-2) s y$ at $\left(\frac{2 s}{p}\right)^{\frac{1}{p-2}}$.

- For $\lambda=0$, apart from the trivial solution $y=0$, we obtain the solutions $t \mapsto s^{\frac{1}{p-2}} \cosh \left(\frac{2 \sqrt{s}}{n-2}(t+\right.$ $c))^{-\frac{n-2}{2}}, c \in \mathbb{R}$, which are positive solutions defined on $\mathbb{R}$, symmetric about their maximum $t=-c$, with $s^{\frac{1}{p-2}}$ as maximum value, and which tend to 0 at infinity.

- For $\lambda \in \mathbb{R}_{-}^{\times}$, we obtain as above a solution which explodes on both sides in finite time.

This shows 2) and concludes the proof.

Corollary 3.13 Let a spacetime $\left(M^{n}, g\right)$ be conformally equivalent to the product $\left(I \times \Sigma,-d t^{2} \oplus\right.$ $\left.g_{\Sigma}\right)$ of an open interval $I \subset \mathbb{R}$ with a closed Riemannian manifold $\left(\Sigma, g_{\Sigma}\right)$ of constant negative scalar curvature. Then there exist infinitely many non-homothetic conformal metrics with constant negative scalar curvature on $\left(M^{n}, g\right)$.

Proof: Immediate consequence of Proposition 3.3 and Lemma 3.12

We turn to the subcritical equation $L_{g_{\Sigma}} u=\lambda u^{p-1}$ on $\Sigma$. First notice that, if $u, v \in C^{\infty}\left(\Sigma, \mathbb{R}_{+}^{\times}\right)$ solve $L_{g_{\Sigma}} u=\lambda u^{p-1}$ and $L_{g_{\Sigma}} v=\mu v^{p-1}$ on $\Sigma$ respectively, for some $\lambda, \mu \in \mathbb{R}$, then $\lambda$ and $\mu$ have the same sign $(\lambda \mu \geq 0$ and vanishes if and only if $\lambda=\mu=0)$ : by formal self-adjointness of $L_{g_{\Sigma}}$,

$$
\lambda \int_{\Sigma} u^{p-1} v d \sigma=\int_{\Sigma}\left(L_{g_{\Sigma}} u\right) v d \sigma=\int_{\Sigma} u\left(L_{g_{\Sigma}} v\right) d \sigma=\mu \int_{\Sigma} u v^{p-1} d \sigma .
$$

In particular, we only need consider uniqueness of solutions to $L_{g_{\Sigma}} u=\lambda u^{p-1}$ with constant $\lambda$ of the same sign as $\mu_{1}\left(L_{g_{\Sigma}}\right)$.

Theorem 3.14 Let $\left(\Sigma^{n-1}, g_{\Sigma}\right)$ be a closed connected Riemannian manifold, where $n \geq 3$. Let $\mu_{1}\left(L_{g_{\Sigma}}\right) \in \mathbb{R}$ be the smallest eigenvalue of $L_{g_{\Sigma}}$ and $p:=\frac{2 n}{n-2}$.

1) If $\mu_{1}\left(L_{g_{\Sigma}}\right)<0$, then for any $S_{\bar{g}} \in \mathbb{R}_{-}^{\times}$the equation $L_{g_{\Sigma}} \varphi=a_{n} S_{\bar{g}} \varphi^{p-1}$ admits a unique smooth positive solution on $\Sigma$.

2) If $\mu_{1}\left(L_{g_{\Sigma}}\right)=0$, then the equation $L_{g_{\Sigma}} \varphi=0$ admits a unique smooth positive solution up to scale on $\Sigma$.

3) For any $\Lambda \in \mathbb{R}_{+}^{\times}$the set

$$
S_{\Lambda}:=\left\{u \in C^{\infty}\left(\Sigma, \mathbb{R}_{+}\right)\left|L_{g_{\Sigma}} u=\lambda u^{p-1},\right| \lambda \mid \leq \Lambda,\|u\|_{L^{p}(\Sigma)} \leq \Lambda\right\}
$$

is compact in $C^{2}(\Sigma, \mathbb{R})$. 
Proof: By Courant's nodal domain theorem, $\operatorname{Ker}\left(L_{g_{\Sigma}}-\mu_{1}\left(L_{g_{\Sigma}}\right)\right)$ is a real line generated by a positive smooth function on $\Sigma$. This already implies 2). Statement 1) relies on the method of sub- and supersolutions developed in [17, 18] and further in [27, 26. We briefly recall the concepts and statements we need for the proof. Given a $C^{1}$ function $f: \Sigma \times \mathbb{R} \rightarrow \mathbb{R}$, a strong sub- (resp. super-) solution for the equation $\Delta u=f(x, u)$ is a $C^{2}$-function $v$ on $\Sigma$ with $\Delta v \leq f(x, v)$ (resp. $\left.\Delta v \geq f(x, v)\right)$ on $\Sigma$. A weak sub- (resp. super-) solution for the equation $\Delta u=f(x, u)$ is a $v \in H^{1,2}(\Sigma) \cap C^{0}(\Sigma, \mathbb{R})$ satisfying $\int_{\Sigma}\left(g_{\Sigma}(d v, d \varphi)-f(x, v) \varphi\right) d \sigma \leq 0\left(\operatorname{resp} . \int_{\Sigma}\left(g_{\Sigma}(d v, d \varphi)-f(x, v) \varphi\right) d \sigma \geq 0\right)$ for all $\varphi \in$ $C^{\infty}\left(\Sigma, \mathbb{R}_{+}\right)$. Of course, every strong sub- or super-solution is a weak one. The steps in the proof of statement 1) are the following:

a) If $v_{1}, v_{2} \in C^{2}(\Sigma, \mathbb{R})$ are strong super-solutions to $\Delta u=f(x, u)$, then $\min \left(v_{1}, v_{2}\right) \in H^{1,2}(\Sigma) \cap$ $C^{0}(\Sigma, \mathbb{R})$ is a weak super-solution to the same equation [26, Prop. 1].

b) Let $v_{1}, v_{2} \in C^{2}(\Sigma, \mathbb{R})$ (resp. $\quad v_{-} \in C^{2}(\Sigma, \mathbb{R})$ ) be strong super-solutions (resp. a strong sub-solution) to $\Delta u=f(x, u)$ with $v_{-} \leq \min \left(v_{1}, v_{2}\right)$. Then there exists a strong solution $v \in C^{2}(\Sigma, \mathbb{R})$ to the same equation with $v_{-} \leq v \leq \min \left(v_{1}, v_{2}\right)$, compare e.g. [15, Thm. 7.4.1] or [17, Lemma 2.6] and references therein.

Now let $u_{1}, u_{2} \in C^{\infty}\left(\Sigma, \mathbb{R}_{+}^{\times}\right)$both solve $L_{g_{\Sigma}} u_{i}=\lambda u_{i}^{p-1}$ for some $\lambda \in \mathbb{R}_{-}^{\times}$. Up to multiplying $u_{1}$ and $u_{2}$ by a positive constant, we may assume that $\lambda=-1$. We construct suitable suband super-solutions for $L_{g_{\Sigma}} w=-w^{p-1}$ in order to be able to assume $u_{1} \leq u_{2}$, compare [26, Lemma 1]. First, if $u \in \operatorname{Ker}\left(L_{g_{\Sigma}}-\mu_{1}\left(L_{g_{\Sigma}}\right)\right)$ is positive, then there is a strong sub-solution to $L_{g_{\Sigma}} w=-w^{p-1}$ of the form $u_{-}:=\alpha u$ with appropriate $\alpha \in \mathbb{R}_{+}^{\times}$: namely $L_{g_{\Sigma}} u_{-} \leq-u_{-}^{p-1}$ if and only if $\alpha \mu_{1}\left(L_{g_{\Sigma}}\right) u \leq-\alpha^{p-1} u^{p-1}$, i.e., if and only if $\alpha \leq \frac{1}{\max _{\Sigma}(u)}\left(-\mu_{1}\left(L_{g_{\Sigma}}\right)\right)^{\frac{1}{p-2}}$ (recall that $\left.\mu_{1}\left(L_{g_{\Sigma}}\right)<0\right)$, whose r.h.s. is positive since $\Sigma$ is compact. Therefore $u_{-}=\alpha u$ is a strong subsolution to $L_{g_{\Sigma}} w=-w^{p-1}$ for $\alpha>0$ sufficiently small. Again, by compactness of $\Sigma$ and continuity of $u_{1}, u_{2}$, one may choose $\alpha>0$ small enough such that $u_{-} \leq u_{i}, i=1,2$. So we are in the situation where $u_{-}$is a strong sub-solution and $u_{1}, u_{2}$ are strong (super-)solutions to $L_{g_{\Sigma}} w=-w^{p-1}$ with $u_{-} \leq \min \left(u_{1}, u_{2}\right)$. By b) just above, there exists a strong solution $v \in C^{2}(\Sigma, \mathbb{R})$ to $L_{g_{\Sigma}} w=-w^{p-1}$ with $u_{-} \leq v \leq \min \left(u_{1}, u_{2}\right)$, in particular $v>0$ on $\Sigma$. Actually, classical elliptic regularity yields $v \in C^{\infty}\left(\bar{\Sigma}, \mathbb{R}_{+}^{\bar{x}}\right)$. As a consequence, for both $i=1,2$,

$$
-\int_{\Sigma} u_{i}^{p-1} v d \sigma=\int_{\Sigma}\left(L_{g_{\Sigma}} u_{i}\right) v d \sigma=\int_{\Sigma} u_{i}\left(L_{g_{\Sigma}} v\right) d \sigma=-\int_{\Sigma} u_{i} v^{p-1} d \sigma,
$$

so that $\int_{\Sigma} u_{i} v\left(u_{i}^{p-2}-v^{p-2}\right) d \sigma=0$. Because of $p-2>0$, we have $u_{i}^{p-2}-v^{p-2} \geq 0$ and therefore $u_{i}^{p-2}-v^{p-2}=0$, that is, $u_{i}=v$ for $i=1,2$, in particular $u_{1}=u_{2}$. This proves statement 1$)$.

The compactness of the set $S_{\Lambda}$ relies mainly on the following so-called regularity theorem (actually needed for the proof of Theorem 3.4, see e.g. [20, Thm. 4.1] or [2, Satz 2.3.3]:

Let $\left(\Sigma^{n-1}, g_{\Sigma}\right)$ be a closed Riemannian manifold with $n \geq 3, p \in\left[2, \infty\left[, h \in C^{\infty}(\Sigma, \mathbb{R})\right.\right.$ and $L:=\Delta+h$. Then for any $\Lambda_{1}, \Lambda_{2} \geq 0$ and $\left.r \in\right] \frac{n-1}{2}(p-2), \infty[$, there exists a constant $C=$ $C\left(\Sigma, g_{\Sigma},\|h\|_{L^{\infty}(\Sigma)}, \Lambda_{1}, \Lambda_{2}, r\right) \geq 0$ and $\left.\alpha=\alpha(r) \in\right] 0,1[$ such that for all almost everywhere nonnegative $\varphi \in H^{1,2}(\Sigma) \cap L^{r}(\Sigma)$ solving (weakly) $L \varphi=\lambda \varphi^{p-1}$ with $|\lambda| \leq \Lambda_{1}$ and $\|\varphi\|_{L^{r}(\Sigma)} \leq \Lambda_{2}$, we have: $\varphi \in C^{\infty}(\Sigma, \mathbb{R})$, either $\varphi>0$ or $\varphi=0$ everywhere on $\Sigma$ and $\|\varphi\|_{C^{2, \alpha}(\Sigma)} \leq C$.

Fixing $r=p=\frac{2 n}{n-2}$ and noticing that $p>\frac{n-1}{2}(p-2)$, the regularity theorem provides, for any $\Lambda \in] 0, \infty[$, the existence of an $\alpha \in] 0,1\left[\right.$ and of a constant $C=C\left(\Sigma, g_{\Sigma}, \Lambda\right)>0$ with $\|\varphi\|_{C^{2, \alpha}(\Sigma)} \leq C$ for all $\varphi \in S_{\Lambda}$. With other words, $S_{\Lambda}$ is included in the closed $C$-ball around the origin in $C^{2, \alpha}(\Sigma, \mathbb{R})$. But by Arzelà-Ascoli theorem, the inclusion $C^{2, \alpha}(\Sigma, \mathbb{R}) \hookrightarrow C^{2}(\Sigma, \mathbb{R})$ is compact, so 
that $S_{\Lambda}$ is relatively compact in $C^{2}(\Sigma, \mathbb{R})$. Thus it remains to show that $S_{\Lambda}$ is closed in $C^{2}(\Sigma, \mathbb{R})$. Consider the map

$$
\Phi: C^{2}\left(\Sigma, \mathbb{R}_{+}\right) \times[-\Lambda, \Lambda] \rightarrow C^{0}(\Sigma, \mathbb{R}), \quad(u, \lambda) \mapsto L_{g_{\Sigma}} u-\lambda u^{p-1} .
$$

We show that $\Phi$ is continuous w.r.t. the standard topologies on both sides. Let $\left(u_{k}, \lambda_{k}\right)_{k \in \mathbb{N}}$ be a sequence of $C^{2}\left(\Sigma, \mathbb{R}_{+}\right) \times[-\Lambda, \Lambda]$ converging to some $(u, \lambda) \in C^{2}\left(\Sigma, \mathbb{R}_{+}\right) \times[-\Lambda, \Lambda]$, i.e., $u_{k} \underset{k \rightarrow \infty}{\longrightarrow} u$ in $C^{2}(\Sigma)$ and $\lambda_{k} \underset{k \rightarrow \infty}{\longrightarrow} \lambda$ in $\mathbb{R}$. Then $\Delta u_{k} \underset{k \rightarrow \infty}{\longrightarrow} \Delta u$ in $C^{0}(\Sigma)$ and, because of $\left\|S_{g_{\Sigma}}\right\|_{C^{0}(\Sigma)}<\infty$, we have $L_{g_{\Sigma}} u_{k} \underset{k \rightarrow \infty}{\longrightarrow} L_{g_{\Sigma}} u$ in $C^{0}(\Sigma)$. Moreover, since $u_{k} \underset{k \rightarrow \infty}{\longrightarrow} u$ in $C^{0}(\Sigma)$, we can fix a small $\varepsilon>0$ and use $\sup _{x \in\left[0,\|u\|_{C^{0}(\Sigma)}+\varepsilon\right]}(p-1) x^{p-2}<\infty$ to deduce that $\left\|u_{k}^{p-1}-u^{p-1}\right\|_{C^{0}(\Sigma)} \leq c \cdot\left\|u_{k}-u\right\|_{C^{0}(\Sigma)}$ for some constant $c>0$ (independent of $k$ ) and all sufficiently large $k \in \mathbb{N}$, in particular $\| u_{k}^{p-1}-$ $u^{p-1} \|_{C^{0}(\Sigma)} \underset{k \rightarrow \infty}{\longrightarrow} 0$. Therefore, $L_{g_{\Sigma}} u_{k}-\lambda_{k} u_{k}^{p-1} \underset{k \rightarrow \infty}{\longrightarrow} L_{g_{\Sigma}} u-\lambda u^{p-1}$, i.e., $\Phi\left(u_{k}, \lambda_{k}\right) \underset{k \rightarrow \infty}{\longrightarrow} \Phi(u, \lambda)$ in $C^{0}(\Sigma)$. Hence $\Phi$ is continuous and thus $\Phi^{-1}(\{0\})$ is closed in $C^{2}\left(\Sigma, \mathbb{R}_{+}\right) \times[-\Lambda, \Lambda]$. But $[-\Lambda, \Lambda]$ being compact, the first projection $\operatorname{pr}_{1}\left(\Phi^{-1}(\{0\})\right)$ of $\Phi^{-1}(\{0\})$ is also closed in $C^{2}\left(\Sigma, \mathbb{R}_{+}\right)$. By restriction, $S_{\Lambda}=\operatorname{pr}_{1}\left(\Phi^{-1}(\{0\})\right) \cap\left\{\varphi \in C^{2}(\Sigma, \mathbb{R}) \mid\|\varphi\|_{L^{p}(\Sigma)} \leq \Lambda\right\}$ is closed in $C^{2}\left(\Sigma, \mathbb{R}_{+}\right) \cap$ $\left\{\varphi \in C^{2}(\Sigma, \mathbb{R}) \mid\|\varphi\|_{L^{p}(\Sigma)} \leq \Lambda\right\}$. Now the set $C^{2}\left(\Sigma, \mathbb{R}_{+}\right) \cap\left\{\varphi \in C^{2}(\Sigma, \mathbb{R}) \mid\|\varphi\|_{L^{p}(\Sigma)} \leq \Lambda\right\}$ is closed in $C^{2}(\Sigma, \mathbb{R})$ : the subset $C^{2}\left(\Sigma, \mathbb{R}_{+}\right)$is obviously closed in $C^{2}(\Sigma, \mathbb{R})$ and, if $u_{k} \underset{k \rightarrow \infty}{\longrightarrow} u$ in $C^{2}(\Sigma, \mathbb{R})$, then also in $C^{0}(\Sigma, \mathbb{R})$ and hence in $L^{p}(\Sigma)$, in particular $\|\cdot\|_{L^{p}(\Sigma)}: C^{2}(\Sigma, \mathbb{R}) \rightarrow \mathbb{R}_{+}$is continuous. On the whole, $S_{\Lambda}$ is closed in $C^{2}(\Sigma, \mathbb{R})$ and therefore compact by the above argument. This shows statement 3 ) and concludes the proof of Theorem 3.14

As for the Riemannian Yamabe problem, uniqueness need not hold in case $\mu_{1}\left(L_{g_{\Sigma}}\right)>0$, as the following example shows, compare [31, pp. 132-135].

Example 3.15 Let $\Sigma^{n-1}:=\Sigma_{1}^{n-2} \times \mathbb{S}^{1}(L)$ be endowed with the product metric $g_{\Sigma}=g_{1} \oplus d t^{2}$, where $\left(\Sigma_{1}^{n-2}, g_{1}\right)$ is a closed Riemannian manifold of constant positive scalar curvature $S_{g_{1}}$ and $\mathbb{S}^{1}(L)$ is the circle of length $L>0$. The subcritical equation $L_{g_{\Sigma}} \varphi=a_{n} S_{\bar{g}} \varphi^{p-1}$ with $S_{\bar{g}} \in \mathbb{R}_{+}^{\times}$can be rewritten in the form

$$
-\frac{\partial^{2} \varphi}{\partial t^{2}}+\Delta_{g_{1}} \varphi+a_{n} S_{g_{1}} \varphi=a_{n} S_{\bar{g}} \varphi^{p-1}
$$

where $\Delta_{g_{1}}: C^{\infty}\left(\Sigma_{1}, \mathbb{R}\right) \rightarrow C^{\infty}\left(\Sigma_{1}, \mathbb{R}\right)$ is the scalar Laplace operator of $\left(\Sigma_{1}, g_{1}\right)$. Looking for solutions of the form $\varphi=y \in C^{\infty}\left(\mathbb{S}^{1}, \mathbb{R}_{+}^{\times}\right)$, we have to find $\frac{L}{k}$-periodic solutions to the ODE $-y^{\prime \prime}+a_{n} S_{g_{1}} y=a_{n} S_{\bar{g}} y^{p-1}$ on $\mathbb{R}$, for any $k \in \mathbb{N} \backslash\{0\}$. Up to multiplying $y$ with a positive constant, we may assume that $a_{n} S_{\bar{g}}=\frac{p}{2}$, so that the ODE becomes $y^{\prime \prime}=s y-\frac{p}{2} y^{p-1}$, where $s:=a_{n} S_{g_{1}} \in \mathbb{R}_{+}^{\times}$. Now Lemma 3.12 states that, for any $\left.T \in\right] \frac{2 \pi}{\sqrt{(p-2) s}}, \infty[$, there exists a $T$-periodic (non-constant) positive solution to $y^{\prime \prime}=s y-\frac{p}{2} y^{p-1}$. Hence, if $\left.L \in\right] \frac{2 \pi}{\sqrt{(p-2) s}}, \infty[$, then there exists a non-constant $L$-periodic positive solution to that equation. More precisely, if $L \in] \frac{2 k \pi}{\sqrt{(p-2) s}}, \frac{2(k+1) \pi}{\sqrt{(p-2) s}}[$ for some $k \in \mathbb{N} \backslash\{0\}$, then there are positive solutions with periods $L, \frac{L}{2}, \ldots, \frac{L}{k}$ respectively to that equation. In particular, the subcritical equation on $\Sigma_{1}^{n-2} \times \mathbb{S}^{1}(L)$ has more than one solution for $L>0$ sufficiently large. Combined with Proposition 3.3 , this fact in turn implies the existence of non-homothetic conformal metrics with constant positive scalar curvature on any spacetime conformally equivalent to $\left(I \times \Sigma,-d t^{2} \oplus g_{\Sigma}\right)$ for $\Sigma$ as above.

However, if the Ricci curvature of $\left(\Sigma, g_{\Sigma}\right)$ is large enough, then uniqueness for the subcritical equation is satisfied: 
Theorem 3.16 (M.-F. Bidaut-Véron \& L. Véron [9]) Let $\left(\Sigma^{n-1}, g_{\Sigma}\right)$ be a closed Riemannian manifold with $n \geq 4$. Assume there exist $\lambda \in \mathbb{R}_{+}^{\times}$and $\left.q \in\right] 2, \infty[$ such that

i) $\operatorname{ric}_{g_{\Sigma}} \geq \frac{n-2}{n-1}(q-2) \lambda \cdot g_{\Sigma}$ and

ii) $q \leq \frac{2(n-1)}{n-3}$

with strict inequality in i) or ii) if $\left(\Sigma^{n-1}, g_{\Sigma}\right)$ is conformally equivalent to $\left(\mathbb{S}^{n-1}\right.$, can). Then the only solution $u>0$ to $\Delta u+\lambda u=u^{q-1}$ is the constant one $u=\lambda^{\frac{1}{q-2}}$.

\section{Examples 3.17}

1. Let $\left(\Sigma^{n-1}, g_{\Sigma}\right)$ be any $n-1(\geq 3)$-dimensional closed Riemannian manifold with constant positive scalar curvature $S_{g_{\Sigma}}$ and ric $_{g_{\Sigma}} \geq \frac{n-2}{n-1} \cdot \frac{S_{g_{\Sigma}}}{n-1} \cdot g_{\Sigma}$. For instance, any Einstein metric - or, more generally, any sufficiently small $C^{2}$-perturbation of an Einstein metric (think e.g. of small perturbations of the round metric on $\mathbb{S}^{2 n+1}$ into Berger metrics) - with constant positive scalar curvature satisfies this condition. Then Theorem 3.16 with $\lambda=a_{n} S_{g_{\Sigma}}$ and $\left.q=p=\frac{2 n}{n-2} \in\right] 2, \frac{2(n-1)}{n-3}$ [ applies and yields in particular the uniqueness of solutions to the subcritical equation $L_{g_{\Sigma}} u=u^{p-1}$ on $\Sigma$.

2. Let $\left(\Sigma^{n-1}, g_{\Sigma}\right):=\left(\Sigma_{1} \times \Sigma_{2}, g_{1} \oplus g_{2}\right)$ with $n_{1}+n_{2}=n-1 \geq 4$ be the Riemannian product of two closed Einstein manifolds with constant positive scalar curvature $S_{g_{1}}$ and $S_{g_{2}}$ respectively. For $\lambda=a_{n} S_{g_{\Sigma}}=\frac{n-2}{4(n-1)}\left(S_{g_{1}}+S_{g_{2}}\right) \in \mathbb{R}_{+}^{\times}$and $\left.q=p=\frac{2 n}{n-2} \in\right] 2, \frac{2(n-1)}{n-3}\left[\right.$, we have $\frac{n-2}{n-1}(q-2) \lambda=$ $\frac{n-2}{(n-1)^{2}}\left(S_{g_{1}}+S_{g_{2}}\right)$. Because of $\operatorname{ric}_{g_{\Sigma}}=\operatorname{ric}_{g_{1}} \oplus \operatorname{ric}_{g_{2}}=\frac{S_{g_{1}}}{n_{1}} g_{1} \oplus \frac{S_{g_{2}}}{n_{2}} g_{2}$, a short computation shows that ric $_{g_{\Sigma}} \geq \frac{n-2}{(n-1)^{2}}\left(S_{g_{1}}+S_{g_{2}}\right) \cdot g_{\Sigma}$ is equivalent to

$$
\frac{n_{2}\left(n_{1}+n_{2}-1\right)}{n_{1}^{2}+n_{1} n_{2}+n_{2}} S_{g_{1}} \leq S_{g_{2}} \leq \frac{n_{1}+n_{1} n_{2}+n_{2}^{2}}{n_{1}\left(n_{1}+n_{2}-1\right)} S_{g_{1}} .
$$

In that case, Theorem 3.16 applies and yields the uniqueness of solutions to the subcritical equation $L_{g_{\Sigma}} u=u^{p-1}$ on $\Sigma$. Note that the inequality just above is in particular fulfilled if the Einstein condition $\frac{S_{g_{1}}}{n_{1}}=\frac{S_{g_{2}}}{n_{2}}$ is.

Note that, on any spacetime of the form $\left(\mathbb{R} \times \Sigma,-d t^{2} \oplus g_{\Sigma}\right)$ with $S_{g_{\Sigma}}$ constant positive and ric $_{g_{\Sigma}} \geq \frac{n-2}{n-1} \cdot \frac{S_{g_{\Sigma}}}{n-1} \cdot g_{\Sigma}$, Lemma 3.12 and Theorem 3.16 imply that the only solutions of the forms $\varphi(t, x)=y(t) \cdot u(x)$ of (2) are constant (in $t$ and $x)$. Still there could exist solutions in non-separated form. For instance, if the group of conformal diffeomorphisms of the spacetime is strictly larger than its isometry group, then there exists a non-constant solution of (2). But, if the spacetime is Einstein and lightlike geodesically complete - e.g. de Sitter spacetime - then there is no nonhomothetic conformal metric which is also Einstein [19, Thm. 1], in particular any conformal transformation is already an isometry.

\section{General case and outlook}

In this section we come back to arbitrary globally hyperbolic spacetimes $\left(M^{n}, g\right)$ with closed Cauchy hypersurface. We face several kinds of problems when looking for a smooth positive global solution to (2). First, we must show the existence of a solution - at least in the weak sense. We have seen that, for standard static spacetimes, we could always reduce the equation to a subcritical eigenvalue problem for the Laplace operator on a spacelike slice, whose solvability is well-known, at 
least in the compact setting. In general, it is possible to fix a spacelike Cauchy hypersurface in $M^{n}$ and to try to solve the Cauchy problem associated to $(2)$ with initial data along the hypersurface. For the case where $M=\mathbb{R}^{4}=\mathbb{R} \times \mathbb{R}^{3}$ with standard Minkowski metric, Konrad Jörgens could show [16] (see also [32, Thm. 6.5]) that, given any $p \in[2,6[$ and any compactly supported smooth initial data on $\mathbb{R}^{3} \simeq\{0\} \times \mathbb{R}^{3}$, there always exists a smooth solution to the Cauchy problem associated to the - slightly different - equation $\square \varphi=-\varphi|\varphi|^{p-2}$. This works in particular for $p=\frac{2 n}{n-2}=4$.

Not much is known for arbitrary globally hyperbolic spacetimes, even with closed Cauchy hypersurface. The subcriticality of the exponent $p=\frac{2 n}{n-2}$ for the embedding of the $H^{1,2}$-Sobolev space of the hypersurface is likely to provide at least weak solutions (in the distributional sense) to (2). The existence of those solutions is tightly connected to the choice of sign for the conformal scalar curvature: which kind of invariant could determine it? It is pointless to try to minimize the energy functional whose critical points are the solutions to the Yamabe problem, for that infimum can be shown to be minus infinity. The regularity of solutions is also an issue in itself, but the really delicate point - also related to the choice of conformal scalar curvature - consists in controlling their sign. For we have no maximum principle available to show that a given solution must be positive. In the particular case of standard static spacetimes, the integration of a given solution (possibly against a particular positive function) along the leaves of the standard foliation by Cauchy hypersurfaces leads to an ordinary differential equation or inequation, that straightforwardly provides obstructions for the existence of positive solutions: if the leafwise integral of a function is negative, then the function itself is negative somewhere.

In general, we cannot expect such an elementary obstruction to the existence of positive solutions, already because no separation of variables is possible. In fact, we first of all have to split the spacetime appropriately, or equivalently, choose a "good" temporal function. There is no canonical choice of temporal function on a given globally hyperbolic spacetime, though some choices are better adapted than other according to the question under consideration, see e.g. [24, 22]. Besides fixing a temporal function, we also have to choose a background metric in the given conformal class. Both choices are intimately connected.

When focussing on the Yamabe equation (2), one could start with an arbitrary splitting $\left(M^{n}, g\right)=$ $\left(\mathbb{R} \times \Sigma,-\beta d t^{2} \oplus g_{t}\right)$ as in Theorem 2.2 and, up to changing the metric $g$ conformally, assume that $\beta=1$. The first and superficial reason for this is that it makes the expression of the d'Alembert operator $\square$ relatively simple, see Lemma 2.3. But this is not necessarily the best choice, as we have already seen: for warped product spacetimes $\left(I \times \Sigma,-d t^{2} \oplus b(t)^{2} g_{\Sigma}\right)$, the choice $b(t)^{-2} g$ of conformal metric leads to the even simpler setting of standard static spacetimes, where the Yamabe problem can be completely solved. Still fixing the splitting $\left(M^{n}, g\right)=\left(\mathbb{R} \times \Sigma,-\beta d t^{2} \oplus g_{t}\right)$, it is elementary to find a metric conformal to $g$ such that all hypersurfaces $\{t\} \times \Sigma$ are maximal, i.e., $\operatorname{tr}_{g_{t}}\left(\frac{\partial g_{t}}{\partial t}\right)=0$ - in particular $\frac{\partial}{\partial t}\left(d \sigma_{g_{t}}\right)=0$, which is the case for Lorentzian products; and a conformal metric such that $\operatorname{tr}_{g_{t}}\left(\frac{\partial g_{t}}{\partial t}\right)=\frac{1}{\beta} \frac{\partial \beta}{\partial t}$, which makes the first-order- $\frac{\partial}{\partial t}$-term in $\square_{g}$ vanish. Each of those choices presents technical advantages as well as drawbacks and we have for the moment no clue about which one could be "best" adapted to the Yamabe equation.

Note that one could also construct for each $t$ a metric with constant scalar curvature in the conformal class of $g_{t}$ on the Cauchy hypersurface $\Sigma$ - which is possible by the existence of a solution to the Riemannian Yamabe problem. But this does not help much in our setting: even assuming the existence of a smooth ${ }^{1} f: I \times \Sigma \longrightarrow \mathbb{R}_{+}^{\times}$such that $f(t, \cdot)^{2} g_{t}=\check{g}_{0}$ does not depend on $t$ and has

\footnotetext{
${ }^{1}$ The smooth dependence of $f$ in $t \in I$ is already a very delicate question, at least in the case of positive Yamabe invariants on $\Sigma$, see e.g. [12].
} 
constant scalar curvature, a metric of the form $-f^{2} d t^{2} \oplus \check{g}_{0}$ is in general not conformally equivalent to a (standard) static one - unless $f$ is constant.

On the whole, the Lorentzian Yamabe problem remains widely open.

Acknowledgements: First and foremost, we thank Christian Bär for drawing our attention to the Lorentzian Yamabe problem. It is also a pleasure to thank all the colleagues whose broad knowledge about Lorentzian geometry and/or partial differential equations enriched our work in a crucial way, particularly Bernd Ammann, Gilles Carron, Piotr Chruściel, Farid Madani, Olaf Müller and Marc Nardmann.

\section{References}

[1] T. Barbot, Globally hyperbolic flat space-times, J. Geom. Phys. 53 (2005), no. 2, 123-165.

[2] C. Bär, Geometrische Analysis, lecture notes, Universität Potsdam, 2011, available at http://geometrie.math. uni-potsdam.de/.

[3] C. Bär, N. Ginoux and F. Pfäffle, Wave equations on Lorentzian manifolds and quantization, ESI Lectures in Mathematics and Physics, EMS Publishing House, 2007.

[4] J.K. Beem, P.E. Ehrlich and K.L. Easley, Global Lorentzian geometry, Second edition, Monographs and Textbooks in Pure and Applied Mathematics 202, Marcel Dekker, 1996.

[5] A.N. Bernal and M. Sánchez, Smoothness of time functions and the metric splitting of globally hyperbolic spacetimes, Comm. Math. Phys. 257 (2005), 43-50.

[6] A.N. Bernal and M. Sánchez, Further results on the smoothability of Cauchy hypersurfaces and Cauchy time functions, Lett. Math. Phys. 77 (2006), no. 2, 183-197.

[7] A.N. Bernal and M. Sánchez, Globally hyperbolic spacetimes can be defined as "causal" instead of "strongly causal", Classical Quantum Gravity 24 (2007), no. 3, 745-749.

[8] A.L. Besse, Einstein manifolds, Ergebnisse der Mathematik und ihrer Grenzgebiete, Springer, 1986.

[9] M.-F. Bidaut-Véron and L. Véron, Nonlinear elliptic equations on compact Riemannian manifolds and asymptotics of Emden equations, Invent. Math. 106 (1991), no. 3, 489-539.

[10] J.T. Burns, Curvature functions on Lorentz 2-manifolds, Pacific J. Math. 70 (1977), 325-335.

[11] Y. Choquet-Bruhat and R. Geroch, Global aspects of the Cauchy problem in general relativity, Comm. Math. Phys. 14 (1969), 329-335.

[12] O. Druet, La notion de stabilité pour des équations aux dérivées partielles elliptiques, Ensaios Matemáticos 19, Soc. Bras. Mat., 2010.

[13] N. Ginoux, Linear wave equations, in: C. Bär et K. Fredenhagen (eds.): "Quantum field theory on curved spacetimes", Lecture Notes in Physics 786 (2009), 59-84, Springer.

[14] N. Ginoux, Analysis on Kähler and Lorentzian manifolds, Habilitation thesis, Universität Regensburg, 2014.

[15] E. Hebey, Introduction à l'analyse non linéaire sur les variétés, Fondations, Diderot, 1997. 
[16] K. Jörgens, Das Anfangswertproblem im Großen für eine Klasse nichtlinearer Wellengleichungen, Math. Z. 77 (1961), 295-308.

[17] J.L. Kazdan and F.W. Warner, Scalar curvature and conformal deformation of Riemannian structure, J. Diff. Geom. 10 (1975), 113-134.

[18] J.L. Kazdan and F.W. Warner, Existence and conformal deformation of metrics with prescribed Gaussian and scalar curvatures, Ann. of Math. (2) 101 (1975), 317-331.

[19] V. Kiosak and V.S. Matveev, There are no conformal Einstein rescalings of complete pseudoRiemannian Einstein metrics, C. R. Math. Acad. Sci. Paris 347 (2009), no. 17-18, 1067-1069.

[20] J.M. Lee and T.H. Parker, The Yamabe problem, Bull. Amer. Math. Soc. (N.S.) 17 (1987), no. $1,37-91$.

[21] E. Minguzzi and M. Sánchez, The causal hierarchy of spacetimes, Recent developments in pseudo-Riemannian geometry, 299-358, ESI Lectures in Mathematics and Physics, EMS Publishing House, 2008; arXiv : gr-qc/0609119.

[22] O. Müller, Special temporal functions on globally hyperbolic manifolds, Lett. Math. Phys. 103 (2013), no. 3, 285-297.

[23] O. Müller and M. Nardmann, ODE-type obstructions to extending prescribed scalar curvature metrics in given conformal classes, in preparation.

[24] O. Müller and M. Sánchez, Lorentzian manifolds isometrically embeddable in $\mathbb{L}^{N}$, Trans. Amer. Math. Soc. 363 (2011), no. 10, 5367-5379.

[25] M. Nardmann, Pseudo-Riemannian metrics with prescribed scalar curvature, $\mathrm{PhD}$ thesis, Universität Leipzig, arXiv:math/0409435.

[26] T. Ouyang, On the positive solutions of semilinear equations $\Delta u+\lambda u+h u^{p}=0$ on compact manifolds. II, Indiana Univ. Math. J. 40 (1991), no. 3, 1083-1141.

[27] T. Ouyang, On the positive solutions of semilinear equations $\Delta u+\lambda u-h u^{p}=0$ on the compact manifolds, Trans. Amer. Math. Soc. 331 (1992), no. 2, 503-527.

[28] M. Sánchez, Structure of Lorentzian tori with a Killing vector field, Trans. Amer. Math. Soc. 349 (1997), no. 3, 1063-1080.

[29] M. Sánchez, Some remarks on causality theory and variational methods in Lorenzian manifolds, Conf. Semin. Mat. Univ. Bari no. 265 (1997); arXiv:0712.0600.

[30] M. Sánchez, On the Geometry of Static Spacetimes, Nonlinear Anal., Theory Methods Appl. 63 (2005), no. 5-7, A, e455-e463; arXiv:math/0406332.

[31] R.M. Schoen, Variational theory for the total scalar curvature functional for Riemannian metrics and related topics, Topics in calculus of variations (Montecatini Terme, 1987), 120154, Lecture Notes in Math. 1365, Springer, 1989.

[32] J. Shatah and M. Struwe, Geometric wave equations, Courant Lecture Notes in Mathematics 2, Amer. Math. Soc., 1998.

[33] M.E. Taylor, Partial differential equations. III. Nonlinear equations, Applied Mathematical Sciences 117, Springer, 1997.

[34] H. Yamabe, On a deformation of Riemannian structures on compact manifolds, Osaka Math. J. 12 (1960), 21-37. 